\title{
Classical Cepheid pulsation models
}

\section{Effects of convection and chemical composition on the period-Iuminosity and period-Wesenheit relations}

\author{
G. Fiorentino ${ }^{1,3}$, M. Marconi ${ }^{2}$, I. Musella ${ }^{2}$, and F. Caputo ${ }^{3}$ \\ 1 INAF - Osservatorio Astronomico di Bologna, via Ranzani 1, 40127 Bologna, Italy \\ e-mail: giuliana.fiorentino@oabo.inaf.it \\ 2 INAF - Osservatorio Astronomico Di Capodimonte, via Moiariello 16, 131 Napoli, Italy \\ e-mail: [marcella;ilaria]@na.astro.it \\ 3 INAF - Osservatorio Astronomico di Roma, via Frascati 33, 00040 Monte Porzio Catone, Italy \\ e-mail: caputo@mporzio.astro.it
}

Received 2 April 2007 / Accepted 8 July 2007

\begin{abstract}
In spite of the relevance of classical Cepheids as primary distance indicators, a general consensus on the dependence of the periodluminosity $(P L)$ relation on the Cepheid chemical composition has not yet been achieved. From the theoretical point of view, our previous investigations were able to reproduce some empirical tests for suitable assumptions on the helium-to-metal relative enrichment, but these results relied on specific assumptions concerning the mass-luminosity relation and the efficiency of the convective transfer in the pulsating envelopes. In this paper, we investigate the effects of the assumed value of the mixing-length parameter $l / H_{\mathrm{p}}$ on the pulsation properties and we release the assumption of a fixed mass-luminosity relation. To this purpose, new nonlinear convective fundamental pulsation models have been computed for various chemical compositions $(Z=0.004,0.008,0.01$ and 0.02$)$ and adopting $l / H_{\mathrm{p}}=1.7-1.8$, which is larger than that (1.5) used in our previous papers. From the extended model set, synthetic $P L$ relations in the various photometric bands are derived using the predicted instability strip together with recent evolutionary tracks. We show that as the $l / H_{\mathrm{p}}$ value increases the pulsation region gets narrower, mostly due to the blueward shift of the red edge for fundamental pulsation, with the effect becoming stronger at the higher metal contents $(Z \geq 0.01)$. However, the comparison of the new models with previously computed models shows that the $l / H_{\mathrm{p}}$ variation has no consequence on the predicted period-Wesenheit $(P W)$ relations, which instead are influenced by the pulsator metal content. On this basis, we present a straightforward way to infer the distance and metal content of variables with observed $B V I$ or $B V K$ magnitudes. As for the $P L$ relations, we show that either the zero-point and the slope are very slightly modified by the $l / H_{\mathrm{p}}$ variation, at constant chemical composition. We also confirm that: (1) moving from visual to longer wavelengths, the predicted period-magnitude distribution for a given metal content becomes narrower and its slope becomes steeper; (2) decreasing the metal content, the $P L$ relations become steeper and brighter, with the amount of this metallicity effect decreasing from optical to near-infrared bands. Overall, we show that our pulsation relations appear fully consistent with the observed properties of Galactic and Magellanic Cloud Cepheids, supporting the predicted steepening and brightening of the $P L$ relations when moving from metal-rich to metal-poor variables. Moreover, we show that the distances inferred by the predicted $P W$ relations agree with recently measured trigonometric parallaxes, whereas they suggest a correction to the values based on the Infrared Surface Brightness technique, as already found from an independent method. Finally, also the pulsation metal contents suggested by the predicted $P W$ relations appear in statistical agreement with spectroscopic $[\mathrm{Fe} / \mathrm{H}]$ measurements.
\end{abstract}

Key words. cosmology: distance scale - stars: variables: Cepheids

\section{Introduction}

Classical Cepheids are the most important distance indicators within the Local Group as well as to external galaxies out to the Virgo cluster. Moreover, they are used to calibrate a host of secondary distance indicators, allowing us to reach cosmological distances and to measure the Hubble constant. However, Cepheid distances are generally derived by adopting "universal" multiband period-luminosity $(P L)$ relations calibrated on the Large Magellanic Cloud (LMC) variables and even in the most recent literature a general consensus on this crucial point has not been achieved.

Regarding the slope of the $P L$ relations, for a long time it has been assumed that it is constant over the total period range, i.e., that the relations are linear. Conversely, recent studies (Tammann \& Reindl 2002; Ngeow \& Kanbur 2004, 2005, 2006;
Sandage et al. 2004; Ngeow et al. 2005) show that in the optical bands the LMC PL relations are not linear, further presenting evidence that the observed data are more consistent with two linear relations and for a discontinuity at $P \sim 10$ days. On the other hand, Persson et al. (2004, hereafter P04) show that the near-infrared $(J H K) P L$ relations of LMC Cepheids with period from $\log P \sim 0.4$ to $\sim 2.0$, are quite linear and remarkably tight, leading Ngeow \& Kanbur (2006) to suggest that the reason for such behavior is because in the sample studied by P04 there are not enough short-period variables.

Indeed, earlier discussions of the nonlinear convective pulsation models computed by our group (Bono et al. 1999b, Paper II; Bono et al. 2000b, Paper III; Caputo et al. 2000, Paper V) have already shown that the instability strip boundaries in the $\log L-\log T_{\mathrm{e}}$ plane are almost linear, but when transformed into the various period-magnitude planes they are 
better described by nonlinear relations, mainly in the optical bands and at the red edge of fundamental pulsation. Consequently, we showed in Paper V that, at fixed metal content: (1) the optical period-magnitude distributions of the predicted pulsators are much better represented by a quadratic line, (2) a discontinuity around $\log P \sim 1.4$ should be adopted to constrain the theoretical results into linear approximations, and (3) the predicted $P L$ relations become more and more linear and tight by moving from optical to near-infrared bands. Those theoretical results have been confirmed by more recent and complete pulsation models (Fiorentino et al. 2002, Paper VIII; Marconi et al. 2005, Paper IX), including also the suggestion that metal-poor $(Z=0.004)$ Cepheids follow $P L$ relations which are steeper and brighter than metal-rich $(Z=0.02)$ ones, with this metallicity effect decreasing from optical to near-infrared bands.

From the observational side, several investigations (e.g., Tammann et al. 2003; Sandage et al. 2004; Groenewegen et al. 2004; Ngeow \& Kanbur 2004) point out the non-universality of the $P L$ relation, but with the Galactic Cepheid $(Z \sim 0.02)$ relations steeper than the LMC $(Z \sim 0.008)$ counterparts. On the other hand, Gieren et al. (2005, hereinafter G05), using the Infrared Surface Brightness method to measure distances, affirm that the $P L$ relation slope is the same for Magellanic and Galactic Cepheids. Moreover, a series of papers from the Araucaria Project (Pietrzynski et al. 2007, and references therein) devoted to the survey of Cepheids in Local Group galaxies suggest no change of the LMC PL slope down to a Cepheid metal abundance of about -1.0 dex. Regarding the zero-point, it has been suggested that metal-rich Cepheids are somehow brighter, at a fixed period, than metal-poor variables (Sasselov et al. 1997; Kennicutt et al. 1998, 2003; Kanbur et al. 2003; Storm et al. 2004, S04; Groenewegen et al. 2004; Sakai et al. 2004; Macri et al. 2006). Although the comparison between theory and observations will be discussed later, we note here that the only two direct tests of the metallicity effect are based on Cepheid observations in the outer and inner fields in M101 (Kennicutt et al. 1998) and NGC 4258 (Macri et al. 2006). These show an oxygen abundance varying from LMClike values $([\mathrm{O} / \mathrm{H}] \sim-0.4$, outer fields) to sovrasolar values $([\mathrm{O} / \mathrm{H}] \sim 0.2-0.3$, inner fields), which is the range where our pulsation models suggest that the sign of the metallicity dependence can be locally reversed. While early linear pulsation models (e.g. Sandage et al. 1999; Alibert et al. 1999; Baraffe \& Alibert 2001) predict a moderate metallicity effect, the nonlinear convective models computed by our group (Papers V, VIII and IX and references therein) show that the metallicity effect is not linear over the range $Z=0.004-0.04$ but shows a "turnover" around $Z \sim 0.02$. As discussed in the quoted papers, if the LMC-calibrated $P L$ relations in the $V$ and $I$ bands are used to infer the Cepheid distance, then the distance modulus of variables with $Z<0.008$, which is the typical metal content of LMC Cepheids, requires a positive correction whose amount increases as the metallicity decreases, whereas for variables with $Z \geq 0.02$ the sign and extent of correction depend on the adopted helium-to-metal enrichment ratio $\Delta Y / \Delta Z$. As a consequence, for very metal-rich $(Z \geq 0.03$ or $[\mathrm{O} / \mathrm{H}] \geq+0.2)$ Cepheids the predicted metallicity correction to the LMC-based distance modulus varies from $\sim-0.15 \mathrm{mag}$ to $\sim+0.25 \mathrm{mag}$ as the ratio $\Delta Y / \Delta Z$ increases from 2 to 3.5 . On this ground, we showed that the empirical metallicity correction suggested by Cepheid observations in the two M101 fields may be accounted for, provided that $\Delta Y / \Delta Z \sim 3.5$. We add that recent spectroscopic $[\mathrm{Fe} / \mathrm{H}]$ measurements of Galactic Cepheids (Romaniello et al. 2005) seem to exclude that the visual $P-M_{V}$ relation is independent of the metal content, as well as that the metallicity correction follows the linear relations suggested by the quoted empirical studies. Conversely, better agreement is found with the predicted nonlinear correction given in Paper VIII.

In spite of this promising agreement with observations, we cannot ignore that the $P L$ relations predicted in our previous papers are based on two fundamental assumptions: the pulsation models are computed with a mixing-length parameter $l / H_{\mathrm{p}}=1.5$ and for each mass the luminosity is fixed according to a canonical (i.e., no mass-loss, no convective core overshooting) mass-luminosity $(M L)$ relation. We remind the reader that Classical Cepheids are post red giant branch (RGB) stars crossing the pulsation region of the HR diagram during the characteristic "blue loop" connected with the He-core burning. Since the luminosity of the loop mainly depends on the original mass and chemical composition of the star, the evolutionary models, as computed under specific assumptions, yield $M L$ relation which can be used to fix the luminosity of pulsation models with a given mass and chemical composition.

Regarding the value of the mixing-length parameter, which is a measure of the convection efficiency and is used to close the system of equations describing the dynamical and convective stellar structure, RR Lyrae pulsation models computed by our group (Caputo et al. 2000, Paper V; Marconi et al. 2003; Di Criscienzo et al. 2004a) show that variations of $l / H_{\mathrm{p}}$ lead to variations in the effective temperature of the instability strip boundaries whose amount increase from the blue to the red edge. Specifically, with $l / H_{\mathrm{p}}$ in the range of 1.1 to 2.0 the effective temperatures at the fundamental blue edge (FBE) and fundamental red edge (FRE) vary by $\delta \log T_{\mathrm{e}}(\mathrm{FBE}) \sim 0.02\left(l / H_{\mathrm{p}}-1.5\right)$ and $\delta \log T_{\mathrm{e}}(\mathrm{FRE}) \sim 0.04\left(l / H_{\mathrm{p}}-1.5\right)$, at constant luminosity. Based on these results, we suspect that the Cepheid $P L$ relation, which is bound to the edges of the instability strip, might also depend on the adopted $l / H_{\mathrm{p}}$ value.

On the other hand, specific modelling of the observed light curves of both RR Lyrae stars and classical Cepheids show that hotter variables are well reproduced with $l / H_{\mathrm{p}} \sim 1.5$, whereas variables located toward the red part of the instability strip require $l / H_{\mathrm{p}} \sim 1.8$ (Bono et al. 2000a, 2002; Castellani et al. 2002; Di Criscienzo et al. 2004b; Marconi \& Clementini 2005; Natale et al., in preparation). These results are consistent with current evolutionary models which adopt relatively high (1.92.2) mixing-length values, as calibrated on observations. Thus, in order to investigate the effects of the adopted mixing-length parameter, we have computed new fundamental pulsation models with $Z=0.004,0.008,0.01$ and $0.02, M=5,7,9$ and $11 M_{\odot}$, and $l / H_{\mathrm{p}}=1.7$ and 1.8 .

The comparison of these new models with those previously computed at $l / H_{\mathrm{p}}=1.5$ is presented in Sect. 2 . In this section, we give also the predicted relations involving the reddening insensitive Wesenheit functions, while in Sect. 3 we present synthetic Cepheid populations with selected chemical compositions and discuss the effects of the $l / H_{\mathrm{p}}$ parameter on the multiband $P L$ relations. The comparison between theoretical results and observed Cepheids is presented in Sect. 4, the conclusions close the paper.

\section{The role of the mixing-length parameter}

The new fundamental pulsation models with $l / H_{\mathrm{p}}=1.7$ and 1.8 are listed in Table 1 together with $l / H_{\mathrm{p}}=1.5$ computations presented in previous papers. The input physics and computing procedures have been previously discussed (Bono et al. 1999a, Paper I; Paper III) and will not be repeated here. For 


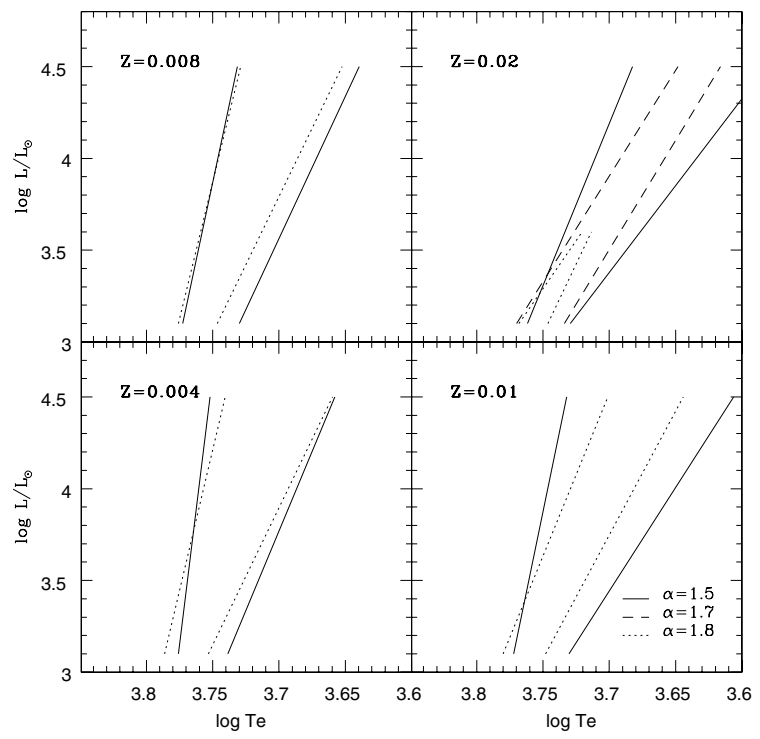

Fig. 1. HR diagram of the fundamental pulsation region when varying the value of the $l / H_{\mathrm{p}}$ parameter as labelled, at four different chemical compositions. The plotted lines are linear interpolations of the computed boundaries.

each given chemical composition and mass, as listed in the first three columns in the Table, the adopted luminosity refers to the canonical $M L$ relation based on Bono et al. (2000c, B00) and Castellani et al. (1992, CCS) evolutionary computations or deals with higher luminosity levels ("over") as produced by convective core overshooting and/or mass loss (see later). The entire pulsation region is covered by varying the model effective temperature $T_{\mathrm{e}}$ by steps of $100 \mathrm{~K}$ and, for the sake of the following discussion, note that increasing (decreasing) by $100 \mathrm{~K}$ the effective temperature of the computed bluest (reddest) model yields nonpulsating structures. Accordingly, we adopt the effective temperature of the computed bluest model, increased by $50 \mathrm{~K}$, as the effective temperature of the FBE, and the effective temperature of the reddest model, decreased by $50 \mathrm{~K}$, as the effective temperature of the FRE. These values, which have the intrinsic uncertainty of $\pm 50 \mathrm{~K}$, are listed in Cols. 7 and 8 in Table 1 .

\subsection{Instability strip and light curves}

Figure 1 shows the HR diagram of the fundamental pulsation region for different assumptions on the $l / H_{\mathrm{p}}$ parameter, at constant chemical composition. The lines depicting the edges of the instability strip are drawn by linear interpolation. We find that the effects of the $l / H_{\mathrm{p}}$ value being increased from 1.5 to 1.8 , are negligible at $Z=0.004$, whereas they become significant when moving to larger metal contents. With $Z=0.01$ the FRE gets bluer by $\sim 300 \mathrm{~K}$ and the FBE redder by $\sim 100 \mathrm{~K}$, while at $Z=0.02$ the effects are even more marked. With $l / H_{\mathrm{p}}=1.7$ the FBE becomes redder by $\sim 100 \mathrm{~K}$ and the FRE bluer by $\sim 300 \mathrm{~K}$. Note that a further increase to $l / H_{\mathrm{p}}=1.8$ yields that the pulsation is completely inhibited for masses larger than $7 M_{\odot}$. This result is due to the higher opacity of metal-rich stellar envelopes which, in turn, produces higher efficiency of convection in damping pulsation.

The bolometric light curves of selected models ${ }^{1}$ with $Z=$ $0.02, Y=0.28$ and with $Z=0.004, Y=0.25$ are presented in

1 All light and radial velocity curves are available upon request to giuliana.fiorentino@oabo.inaf.it.

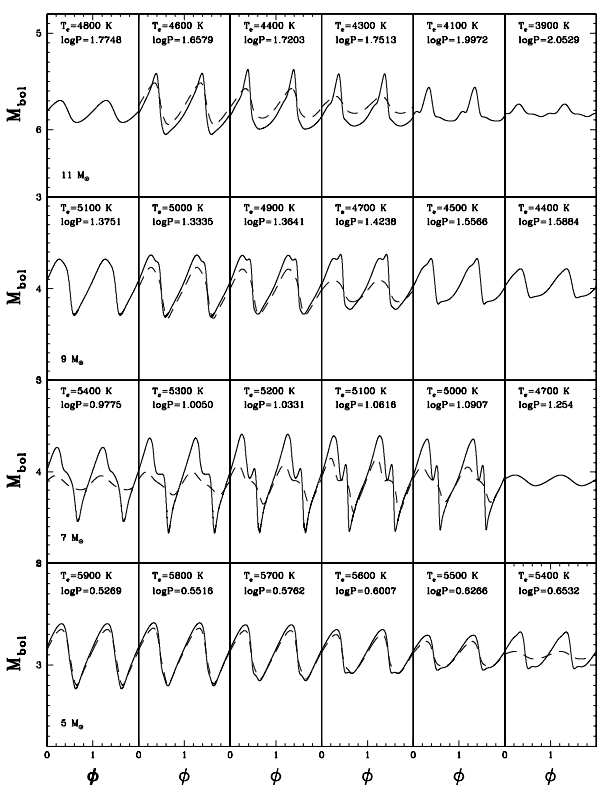

Fig. 2. Effects of varying $l / H_{\mathrm{p}}$ from 1.5 (solid lines) to 1.7 (dashed lines) on fundamental bolometric light curves at $Z=0.02$.

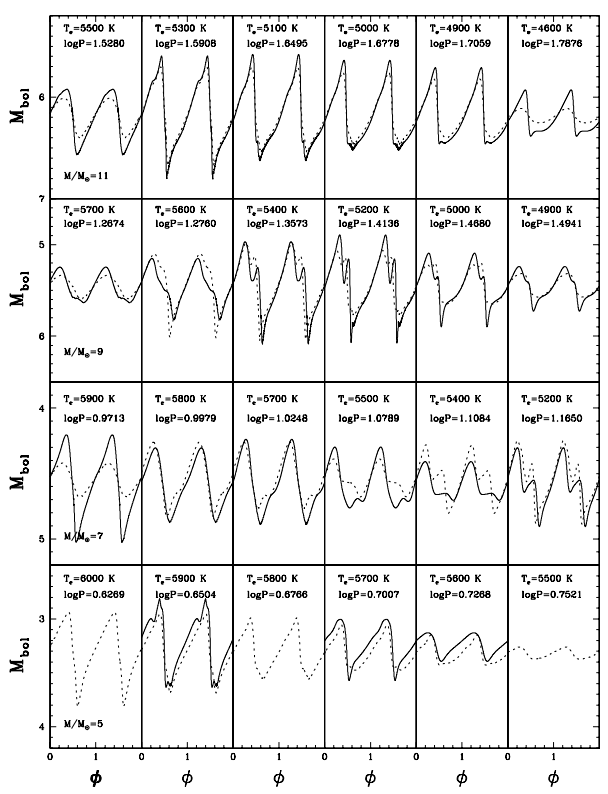

Fig. 3. Effects of varying $l / H_{\mathrm{p}}$ from 1.5 (solid lines) to 1.8 (dashed lines) on fundamental bolometric light curves at $Z=0.004$.

Figs. 2 and 3 respectively. As discussed in Papers I and III, the morphology and the amplitude of the curves vary within the instability strip, depending on the adopted chemical composition, mass and luminosity. In particular, variations of the stellar mass for a fixed $M L$ relation or variations in the $M L$ relation for a fixed mass, affect both the amplitude and the morphology of the light curves (see discussion in Papers I and III). As for the dependence on the mixing length parameter, we notice that by increasing the $l / H_{\mathrm{p}}$ value, the pulsation amplitude and size of the various secondary features decrease. Concerning the Hertzsprung progression (HP), which is the relationship between the pulsation period and the phase of the bump that appears in the light and radial velocity curves for periods around $10 \mathrm{~d}$, we find that in the range $0.004 \leq Z \leq 0.02$ an increase in the metal content causes a 
Table 1. Intrinsic parameters for fundamental pulsating models.

\begin{tabular}{|c|c|c|c|c|c|c|c|c|}
\hline$Z$ & $Y$ & $M / M_{\odot}$ & $\log L / L_{\odot}$ & $M L$ & $l / H_{\mathrm{p}}$ & $T_{\mathrm{e}, \mathrm{FRE}}(\mathrm{K})$ & $T_{\mathrm{e}, \mathrm{FBE}}(\mathrm{K})$ & References \\
\hline 0.004 & 0.250 & 3.50 & $2.61 / 2.86$ & CCS/over & 1.5 & $5800 / 5600$ & $6000 / 6000$ & M04 \\
\hline 0.004 & 0.250 & 4.00 & 2.82 & CCS & 1.5 & 5650 & 5900 & M04 \\
\hline 0.004 & 0.250 & 5.00 & $3.07 / 3.30$ & CCS/over & 1.5 & $5600 / 5400$ & $5900 / 6100$ & Paper I \\
\hline 0.004 & 0.250 & 5.00 & 3.24 & B00 & 1.8 & 5500 & 6000 & This paper \\
\hline 0.004 & 0.250 & 7.00 & $3.65 / 3.85$ & CCS/over & 1.5 & $5200 / 4900$ & $5900 / 5900$ & Paper I \\
\hline 0.004 & 0.250 & 7.00 & 3.73 & B00 & 1.8 & 5200 & 5900 & This paper \\
\hline 0.004 & 0.250 & 7.15 & 3.73 & CCS & 1.5 & 5100 & 5900 & M04 \\
\hline 0.004 & 0.250 & 7.30 & 3.76 & CCS & 1.5 & 5000 & 5900 & M04 \\
\hline 0.004 & 0.250 & 7.45 & 3.79 & CCS & 1.5 & 5000 & 5900 & M04 \\
\hline 0.004 & 0.250 & 9.00 & $4.00 / 4.25$ & CCS/over & 1.5 & $4900 / 4500$ & $5800 / 5700$ & Paper I \\
\hline 0.004 & 0.250 & 9.00 & 4.09 & B00 & 1.8 & 4900 & 5700 & This paper \\
\hline 0.004 & 0.250 & 11.00 & $4.40 / 4.65$ & CCS/over & 1.5 & $4400 / 4700$ & $5500 / 5500$ & Paper I \\
\hline 0.004 & 0.250 & 11.00 & 4.39 & B00 & 1.8 & 4600 & 5500 & This paper \\
\hline 0.008 & 0.250 & 3.50 & $2.57 / 2.78$ & CCS/over & 1.5 & $5800 / 5600$ & $5950 / 5900$ & M04 \\
\hline 0.008 & 0.250 & 4.00 & $2.78 / 3.07$ & CCS/over & 1.5 & $5650 / 5300$ & $5900 / 5900$ & M04 \\
\hline 0.008 & 0.250 & 5.00 & $3.07 / 3.30$ & CCS/over & 1.5 & $5500 / 5300$ & $5900 / 6000$ & Paper I \\
\hline 0.008 & 0.250 & 5.00 & 3.14 & B00 & 1.8 & 5500 & 5900 & This paper \\
\hline 0.008 & 0.250 & 6.55 & 3.55 & CCS & 1.5 & 5100 & 5900 & Paper VI \\
\hline 0.008 & 0.250 & 6.70 & 3.59 & CCS & 1.5 & 5100 & 5900 & Paper VI \\
\hline 0.008 & 0.250 & 6.85 & 3.62 & CCS & 1.5 & 5100 & 5800 & Paper VI \\
\hline 0.008 & 0.250 & 7.00 & $3.65 / 3.85$ & CCS/over & 1.5 & $5000 / 4700$ & $5800 / 5700$ & Paper I \\
\hline 0.008 & 0.250 & 7.00 & 3.62 & B00 & 1.8 & 5200 & 5800 & This paper \\
\hline 0.008 & 0.250 & 7.15 & 3.69 & CCS & 1.5 & 4900 & 5800 & Paper VI \\
\hline 0.008 & 0.250 & 7.30 & 3.72 & CCS & 1.5 & 4900 & 5800 & Paper VI \\
\hline 0.008 & 0.250 & 7.45 & 3.75 & CCS & 1.5 & 4900 & 5800 & Paper VI \\
\hline 0.008 & 0.250 & 9.00 & $4.00 / 4.25$ & CCS/over & 1.5 & $4700 / 4200$ & $5600 / 5300$ & Paper I \\
\hline 0.008 & 0.250 & 9.00 & 3.99 & B00 & 1.8 & 4900 & 5600 & This paper \\
\hline 0.008 & 0.250 & 11.00 & $4.40 / 4.65$ & CCS/over & 1.5 & $4600 / 4200$ & $5200 / 5200$ & Paper I \\
\hline 0.008 & 0.250 & 11.00 & 4.28 & В00 & 1.8 & 4600 & 5400 & This paper \\
\hline 0.01 & 0.260 & 5.00 & 3.13 & B00 & 1.5 & 5300 & 5800 & Paper IX \\
\hline 0.01 & 0.260 & 7.00 & 3.61 & B00 & 1.5 & 4900 & 5900 & Paper IX \\
\hline 0.01 & 0.260 & 9.00 & 3.98 & B00 & 1.5 & 4500 & 5600 & Paper IX \\
\hline 0.01 & 0.260 & 11.00 & 4.27 & B00 & 1.5 & 4200 & 5400 & Paper IX \\
\hline 0.01 & 0.260 & 5.00 & 3.13 & B00 & 1.8 & 5500 & 5900 & This paper \\
\hline 0.01 & 0.260 & 7.00 & 3.61 & B00 & 1.8 & 5200 & 5800 & This paper \\
\hline 0.01 & 0.260 & 9.00 & 3.98 & B00 & 1.8 & 4900 & 5400 & This paper \\
\hline 0.01 & 0.260 & 11.00 & 4.27 & B00 & 1.8 & 4500 & 5100 & This paper \\
\hline 0.02 & 0.250 & 5.00 & 3.00 & B00 & 1.5 & 5300 & 5700 & Paper IX \\
\hline 0.02 & 0.250 & 7.00 & 3.49 & B00 & 1.5 & 4800 & 5600 & Paper IX \\
\hline 0.02 & 0.250 & 9.00 & 3.86 & B00 & 1.5 & 4400 & 5300 & Paper IX \\
\hline 0.02 & 0.250 & 11.00 & 4.15 & B00 & 1.5 & 4300 & 5000 & Paper IX \\
\hline 0.02 & 0.260 & 5.00 & 3.02 & B00 & 1.5 & 5400 & 5800 & Paper IX \\
\hline 0.02 & 0.260 & 7.00 & 3.51 & B00 & 1.5 & 4900 & 5600 & Paper IX \\
\hline 0.02 & 0.260 & 9.00 & 3.88 & B00 & 1.5 & 4600 & 5500 & Paper IX \\
\hline 0.02 & 0.260 & 11.00 & 4.17 & B00 & 1.5 & 4300 & 5300 & Paper IX \\
\hline 0.02 & 0.280 & 4.00 & 2.97 & over & 1.5 & 5300 & 5800 & B01 \\
\hline 0.02 & 0.280 & 4.50 & 2.90 & CCS & 1.5 & 5500 & 5800 & B01 \\
\hline 0.02 & 0.280 & 5.00 & $3.07 / 3.30$ & CCS/over & 1.5 & $5400 / 5400$ & $5900 / 5700$ & Paper I \\
\hline 0.02 & 0.280 & 5.00 & 3.07 & В00 & 1.7 & 5400 & 5900 & This paper \\
\hline 0.02 & 0.280 & 5.00 & 3.07 & B00 & 1.8 & 5550 & 5950 & This paper \\
\hline 0.02 & 0.280 & 6.25 & 3.42 & CCS & 1.5 & 5000 & 5500 & B01 \\
\hline 0.02 & 0.280 & 6.50 & 3.48 & CCS & 1.5 & 5000 & 5600 & B01 \\
\hline 0.02 & 0.280 & 6.75 & 3.54 & CCS & 1.5 & 4900 & 5550 & B01 \\
\hline 0.02 & 0.280 & 7.00 & $3.65 / 3.85$ & CCS/over & 1.5 & $4700 / 4400$ & $5400 / 5200$ & Paper I \\
\hline 0.02 & 0.280 & 7.00 & 3.56 & B00 & 1.7 & 5000 & 5400 & This paper \\
\hline 0.02 & 0.280 & 7.00 & 3.56 & B00 & 1.8 & 5150 & 5350 & This paper \\
\hline 0.02 & 0.280 & 9.00 & $4.00 / 4.25$ & CCS/over & 1.5 & $4400 / 3900$ & $5100 / 4900$ & Paper I \\
\hline 0.02 & 0.280 & 9.00 & 3.92 & B00 & 1.7 & 4700 & 5000 & This paper \\
\hline 0.02 & 0.280 & 11.00 & 4.40 & CCS & 1.5 & 3900 & 4800 & Paper I \\
\hline 0.02 & 0.280 & 11.00 & 4.21 & B00 & 1.7 & 4300 & 4700 & This paper \\
\hline 0.02 & 0.310 & 5.00 & 3.13 & B00 & 1.5 & 5400 & 5900 & Paper VIII \\
\hline 0.02 & 0.310 & 7.00 & 3.62 & B00 & 1.5 & 4900 & 5600 & Paper VIII \\
\hline 0.02 & 0.310 & 9.00 & 3.98 & B00 & 1.5 & 4500 & 5300 & Paper VIII \\
\hline 0.02 & 0.310 & 11.00 & 4.27 & B00 & 1.5 & 4200 & 5100 & Paper VIII \\
\hline
\end{tabular}

B01: Bono et al. (2001); Paper VI: Bono et al. (2000d); M04: Marconi et al. (2004). 


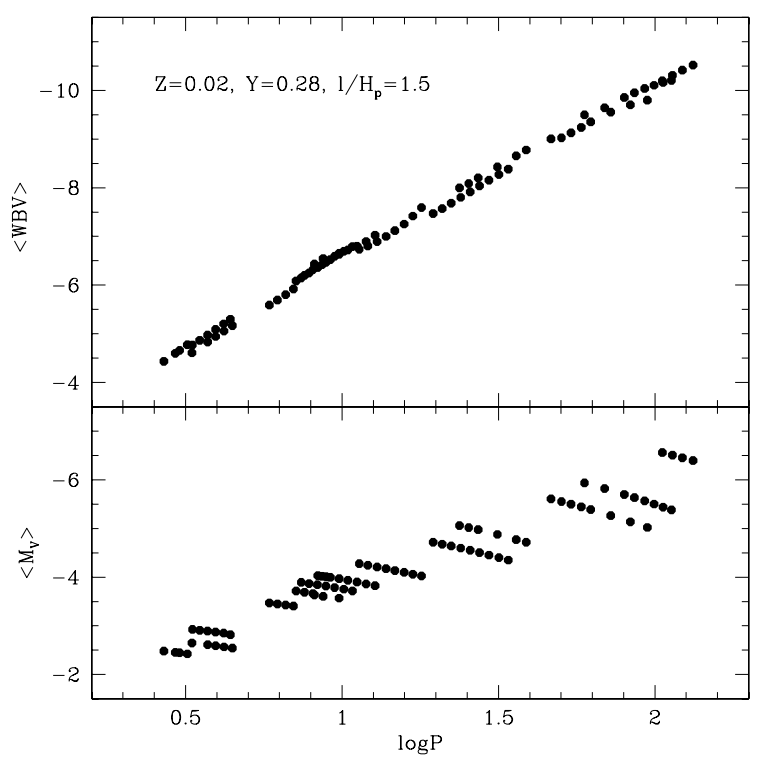

Fig. 4. Absolute visual magnitude $\left\langle M_{V}\right\rangle$ and $W B V$ function versus period for fundamental pulsators with $Z=0.02, Y=0.28$ and $l / H_{\mathrm{p}}=1.5$.

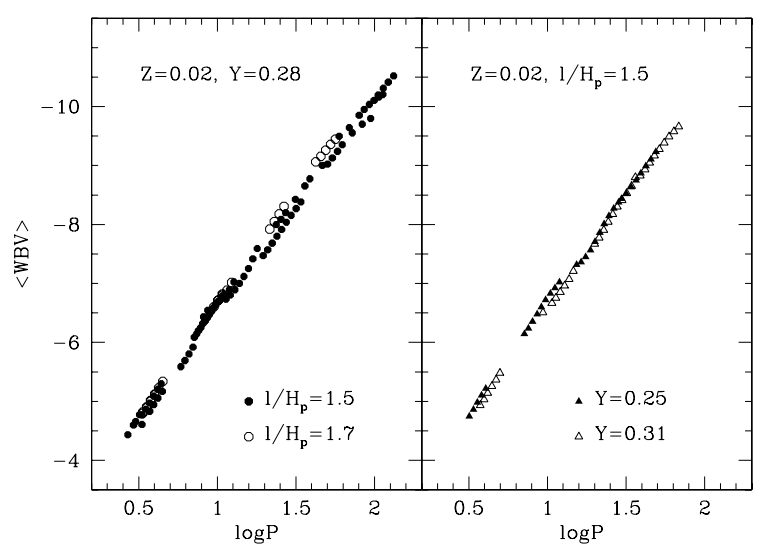

Fig. 5. $W B V$ function versus period for fundamental pulsators with $Z=$ 0.02 , varying the value of the mixing-length parameter (left panel) and the helium content (right panel).

shift of the HP center toward shorter periods (see also Papers VI and IX) for each selected $l / H_{\mathrm{p}}$ value.

\subsection{Predicted period-Wesenheit relations}

The bolometric light curves provided by the non-linear approach have been transformed into the observational bands UBVRIJK by means of the model atmospheres by Castelli et al. (1997a,b). The amplitudes in the various spectral bands and, after a timeintegration, the pulsator predicted magnitude- and intensityaveraged mean magnitudes are derived. In the following, we refer to selected intensity-weighted mean magnitudes $\left\langle M_{i}\right\rangle$ and colors $\left[\left\langle M_{i}\right\rangle-\left\langle M_{j}\right\rangle\right]$, nonetheless the whole set of data, including magnitude-averaged values, are available upon request to the authors.

It is well known that a restatement of the Stefan's law for pulsating stars is the period relation $P=f\left(M, L, T_{\mathrm{e}}\right)$ for which the pulsation period of each given variable is uniquely defined by its mass, bolometric luminosity, and effective temperature. Once transformed into the observational plane, this relation yields that the pulsator absolute magnitude $M_{i}$ in a given photometric

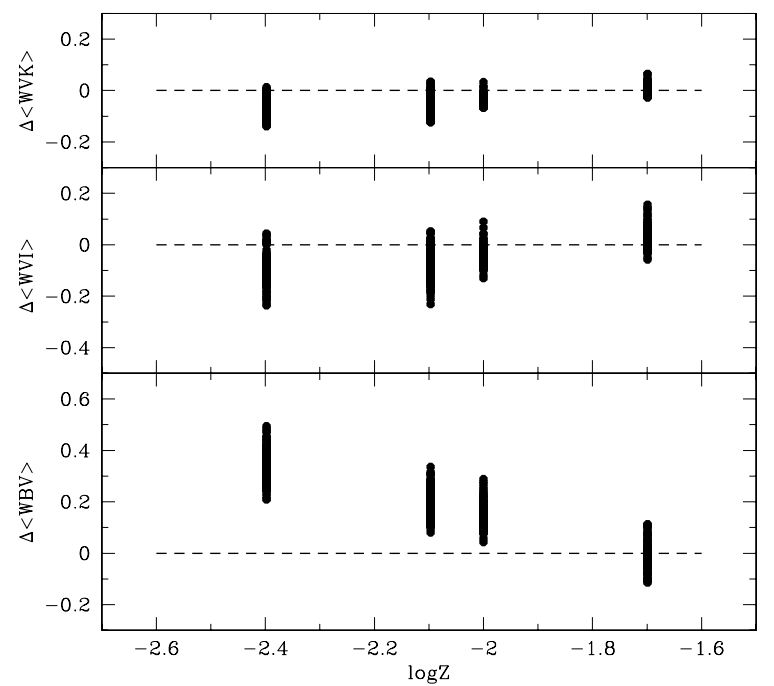

Fig. 6. Residuals of the Wesenheit functions with respect to the $P W$ relations at $Z=0.02, Y=0.28$ and $l / H_{\mathrm{p}}=1.5$.

bandpass is a linear function of the pulsation period, mass ( $M$ in solar units) and color index $\left[\left\langle M_{i}\right\rangle-\left\langle M_{j}\right\rangle\right]$, i.e.,

$M_{i}=a+b \log P+c \log M+d\left[\left\langle M_{i}\right\rangle-\left\langle M_{j}\right\rangle\right]$,

for each given chemical composition. We emphasize that the mass-dependent period-luminosity-color $(P L C)$ relations hold for individual variables and their use together with measured metal contents, absolute magnitudes and intrinsic colors, provides precise values of the "pulsation" mass $M_{\mathrm{p}}$, i.e. the actual mass, of each Cepheid. In the case of Cepheids located at the same distance and with the same reddening and metal content, one can estimate the mass range covered by the variables, and in turn the slope of the empirical $M_{\mathrm{p}} L$ relation, independent of the distance and reddening value (see also Beaulieu et al. 2001).

However, the adoption of the PLC relations requires accurate knowledge of the reddening and for this reason, taking advantage of the evidence that the color coefficients are not too different from the extinction-to-reddening ratios provided by optical and near-infrared reddening laws, the so-named Wesenheit functions have been widely used to bypass interstellar extinction problems (Madore 1982; Madore \& Freedman 1991; Tanvir 1999; Paper V). In this paper, adopting from Dean et al. (1978) and Laney \& Stobie (1993) the absorption to reddening ratios $A_{\lambda} / E(B-V)$

$A_{V}=3.07+0.28(B-V)_{0}+0.04 E(B-V)$

$A_{I}=1.82+0.205(B-V)_{0}+0.022 E(B-V)$

$A_{J}=0.764 ; A_{K}=0.279$

we use ${ }^{2}$ the quantities $W B V=\left\langle M_{V}\right\rangle-3.30\left[\left\langle M_{B}\right\rangle-\left\langle M_{V}\right\rangle\right]$, $W V I=\left\langle M_{V}\right\rangle-2.52\left[\left\langle M_{V}\right\rangle-\left\langle M_{I}\right\rangle\right], W V J=\left\langle M_{V}\right\rangle-1.30\left[\left\langle M_{V}\right\rangle-\right.$ $\left.\left\langle M_{J}\right\rangle\right], W V K=\left\langle M_{V}\right\rangle-1.09\left[\left\langle M_{V}\right\rangle-\left\langle M_{K}\right\rangle\right]$ and $W J K=$ $\left\langle M_{K}\right\rangle-0.575\left[\left\langle M_{J}\right\rangle-\left\langle M_{K}\right\rangle\right]$.

As shown in Fig. 4 for a selected chemical composition, the adoption of the reddening-free Wesenheit functions also has the effect of significantly reducing the dispersion of visual magnitudes at a given period. Moreover, we stress that the $P W$ relations have the undeniable advantage of being linear over the

${ }^{2}$ By artificially reddening our pulsation models, we find $A_{V} / E(B-$ $V)=3.30 \pm 0.05$ and $A_{I} / E(B-V)=1.99 \pm 0.04$ with $E(B-V)$ up to 2 mag. 
Table 2. Predicted mass-dependent $P W$ relations for fundamental pulsators with $Z=0.004-0.02$, as based on intensity-averaged magnitudes of the pulsators. The intrinsic dispersion of the constant term includes the variation of helium content and mixing-length parameter, at constant $Z$. The last column gives the resulting uncertainty $\epsilon_{P W}\left(\log M_{\mathrm{p}}\right)$ on the pulsation mass inferred by these relations.

\begin{tabular}{lccccc}
\hline \hline$W$ & $a$ & $b$ & $c$ & $d$ & $\epsilon_{P W}\left(\log M_{\mathrm{p}}\right)$ \\
\hline \multicolumn{5}{c}{$W=a+b \log P+c \log M_{\mathrm{p}}+d \log Z$} \\
$W B V$ & $-3.52 \pm 0.07$ & $-3.45 \pm 0.02$ & $-1.16 \pm 0.05$ & $-0.67 \pm 0.01$ & 0.06 \\
$W V I$ & $-1.21 \pm 0.05$ & $-2.43 \pm 0.01$ & $-2.66 \pm 0.04$ & $+0.14 \pm 0.01$ & 0.02 \\
$W V J$ & $-0.79 \pm 0.05$ & $-2.30 \pm 0.01$ & $-2.65 \pm 0.04$ & $+0.17 \pm 0.01$ & 0.02 \\
$W V K$ & $-1.08 \pm 0.04$ & $-2.47 \pm 0.01$ & $-2.64 \pm 0.03$ & $+0.15 \pm 0.01$ & 0.02 \\
$W J K$ & $-1.20 \pm 0.04$ & $-2.55 \pm 0.01$ & $-2.55 \pm 0.03$ & $+0.14 \pm 0.01$ & 0.02 \\
\hline
\end{tabular}

whole period range, as already discussed in Paper $\mathrm{V}$ and recently emphasized by Ngeow \& Kanbur (2005) from measurements of LMC Cepheids. Regarding the effects of $l / H_{\mathrm{p}}$ (at fixed $Z$ and $Y$ ) and $Y$ (at fixed $l / H_{\mathrm{p}}$ and $Z$ ), we show in Fig. 5 that they are quite negligible. Conversely, adopting the $P W$ relations at $Z=0.02$, $Y=0.28$ and $l / H_{\mathrm{p}}=1.5$ as reference relations, we show in Fig. 6 that the residuals of all the models listed in Table 1 suggest a metallicity effect in the sense that the $W B V$ quantity becomes fainter whereas $W V I$ and $W V K$ become slightly brighter as the metal content decreases, at constant period.

As a whole, taking the pulsator mass and luminosity as independent parameters, a linear interpolation through all the fundamental models listed in Table 1 yields the mass-dependent $P W$ relations given in Table 2, where the intrinsic dispersion of the constant term includes the variation of $Y$ and $l / H_{\mathrm{p}}$ at constant $Z$. These $P W$ relations cannot be used to obtain distances but are important to estimate, independent of reddening, the Cepheid pulsation mass $M_{\mathrm{p}}$ with the formal uncertainty $\epsilon_{P W}\left(\log M_{\mathrm{p}}\right)$ listed in the last column in this table, once the distance and metal content are known.

We stated that in the framework of canonical (no mass-loss, no convective core overshooting) evolutionary predictions, the bolometric luminosity of a classical Cepheid is mainly governed by the chemical composition and the original, or "evolutionary", stellar mass $M_{\mathrm{e}}$. The properties of central He-burning intermediate-mass stars have been extensively discussed (CCS; B00; Girardi et al. 2000; Castellani et al. 2003, and references therein) and the relevant literature contains several theoretical $M L$ relations which are widely used to estimate the Cepheid evolutionary mass.

In this paper, as reference canonical $M L$ relation in the mass range $4-15 M_{\odot}$, we adopt the B00 relation:

$\log L_{\text {can }}=0.90+3.35 \log M_{\mathrm{e}}+1.36 \log Y-0.34 \log Z$

where mass and luminosity are in solar units. This relation has a standard deviation $\sigma=0.02$ which accounts for both the blueward and the redward portion of the loop, i.e., the 2nd and 3rd crossing $^{3}$, respectively, of the pulsation region. The introduction of an $M L$ relation, which limits the allowed values of mass and luminosity, yields "evolutionary" $P W_{\mathrm{e}}$ relations where the massterm is removed. This procedure has been followed in our previous papers, whereas here we wish to avoid any assumption on the $M L$ relation taking advantage of the various luminosity levels listed in Table 1 for a fixed mass.

Based on the canonical luminosity given by Eq. (2) as a reference level, we estimate the difference $\log L / L_{\text {can }}$ for each pulsation model. Then, by a linear interpolation through all the models we obtain the relations given in Table 3 . According to these relations, once the value of the $L / L_{\text {can }}$ ratio is adopted, where

${ }^{3}$ The first crossing of the instability strip deals with the post-ZAMS evolution towards the red giant branch.
$L_{\text {can }}$ is given by Eq. (2), we can determine the distance to individual Cepheids with the accuracy $\sigma_{W}$ listed in the last column of the table, provided that the Cepheid metal content is known. We emphasize that, aside from minor discrepancies among the canonical $M L$ relations provided by different authors, a variation in luminosity with respect to the reference canonical value can originate in the occurrence of a convective core overshooting or mass-loss before or during the He-burning phase. In the former case, the star is over-luminous by $\log L / L_{\text {can }} \sim 0.20$ for its mass and chemical composition (see also Chiosi et al. 1993), and in the latter, the star has a smaller mass for its luminosity and, depending on the progenitor mass $M_{\mathrm{pr}}$ and the amount of massloss $\Delta M$, one can estimate $\log L / L_{\text {can }} \sim 0.9 \Delta M / M_{\mathrm{pr}}$ (Castellani \& Degl'Innocenti 1995; B00; Castellani et al. 2003). It is important that both cases yield positive $\log L / L_{\mathrm{can}}$ ratios and that the $P W_{\mathrm{e}}$ relations at $L=L_{\mathrm{can}}$ provide the maximum value of the Cepheid distance, with the intrinsic distance modulus decreasing as $\delta \mu_{0}=\gamma \log L / L_{\mathrm{can}}$, where $\gamma$ is the coefficient listed in Table 3 . Finally, we draw attention to the opposite metallicity dependence of the $P-W B V$ relation with respect to the other Wesenheit functions. Such a feature provides a quite straight method to estimate the Cepheid metal content, independent of distance and reddening and with a small effect of the adopted $L / L_{\text {can }}$ ratio (Sect. 4 ).

\section{Synthetic PL relations}

In the previous section, it was shown that the $P W$ relations are quite unaffected by the value of the mixing-length parameter, although the edges of the pulsation region with $Z \geq 0.008$ depend on the adopted $l / H_{\mathrm{p}}$ value. In order to investigate the role of the mixing-length parameter on the $P L$ relations, we populate the predicted instability strip adopting evolutionary computations and using the procedure suggested by Kennicutt et al. (1998) and adopted in our previous papers.

In Papers $\mathrm{V}$ and IX, we derived synthetic $P L$ relations by populating the predicted instability strip according to an assumed mass distribution and adopting, for each mass and chemical composition, the canonical luminosity given by Eq. (2). Here, we present new synthetic $P L$ relations which use evolutionary tracks and take into account also the evolutionary times spent by the Cepheids inside the strip. For this purpose, we adopted the evolutionary computations by Pietrinferni et al. (2004) which cover a mass range from 3 to $10 M_{\odot}$ and a very large metallicity range. Before proceeding, note that the mixing-length parameter used in the evolutionary computations is $l / H_{\mathrm{p}}=1.9$. However, the meaning of this parameter when computing evolutionary sequences is different from that adopted in the pulsation code. The discussion of the differences between the two convective treatments is beyond the scope of this paper and for details we refer interested readers to Bono \& Stellingwerf (1994) and Stellingwerf (1982). 
Table 3. Predicted evolutionary $P W_{\mathrm{e}}$ relations for fundamental pulsators with $Z=0.004-0.02$, as based on intensity-averaged magnitudes of the pulsators. The last column gives the intrinsic dispersion of the relations.

\begin{tabular}{lccccc}
\hline \hline$W$ & $\alpha$ & $\beta$ & $\gamma$ & $\delta$ & $\sigma_{W}$ \\
\hline \multicolumn{5}{c}{$W=\alpha+\beta \log P+\gamma \log L / L_{\mathrm{can}}+\delta \log Z$} \\
$W B V$ & $-4.18 \pm 0.06$ & $-3.84 \pm 0.01$ & $+0.72 \pm 0.02$ & $-0.71 \pm 0.01$ & 0.06 \\
$W V I$ & $-2.67 \pm 0.11$ & $-3.30 \pm 0.01$ & $+0.84 \pm 0.04$ & $+0.08 \pm 0.01$ & 0.11 \\
$W V J$ & $-2.36 \pm 0.12$ & $-3.23 \pm 0.01$ & $+0.86 \pm 0.04$ & $+0.11 \pm 0.01$ & 0.12 \\
$W V K$ & $-2.53 \pm 0.10$ & $-3.33 \pm 0.01$ & $+0.89 \pm 0.04$ & $+0.09 \pm 0.01$ & 0.10 \\
$W J K$ & $-2.61 \pm 0.09$ & $-3.38 \pm 0.01$ & $+0.90 \pm 0.03$ & $+0.08 \pm 0.01$ & 0.10 \\
\hline
\end{tabular}

To build a synthetic population we extract each mass using an initial mass function (IMF) with a power law distribution $\left(P(M) \sim 1 / M^{3}\right)$ and a constant star formation rate. Even if the latter assumption is not reliable for a complex stellar population like LMC (Cioni et al. 2006), our method of populating stellar tracks crossing the IS is an improvement on the classical uniform distribution. By interpolating the evolutionary tracks, we assign both a luminosity and an effective temperature to each synthetic star, and the total number of extractions $(\sim 50000)$ is fixed in order to obtain a number of synthetic stars falling into the instability strip of the order of $10^{3}$, in analogy to our previous procedures. Once a synthetic stellar population is built, we use the constraints of the pulsation theory to reject objects outside the strip and to assign the expected period to each selected fundamental pulsator. To this purpose, we interpolate through the predicted boundary temperatures (FBE and FRE, see Cols. 7 and 8 in Table 1) to select the synthetic stars with FBE $\geq \log T_{\mathrm{e}} \geq$ FRE and we determine the period by means of the $P=f\left(L, M, T_{\mathrm{e}}, Z\right)$ relation provided by a regression through all the fundamental models. Then, using the same atmosphere models adopted in Sect. 2.2, we calculate the pulsator absolute magnitude in the various photometric bands. Left and right panels in Fig. 7 show the results of these simulations at $Z=0.004$ and 0.02 , respectively, adopting $l / H_{\mathrm{p}}=1.5$. It is of interest to note that decreasing the metal content increases the predicted number of faint and hot (i.e., short period) pulsators, fully supporting recent suggestions (Cordier et al. 2003) that explain the occurrence of faint short period Cepheids in the Small Magellanic Cloud $(Z \sim 0.004)$ as an effect of the decreased metallicity.

For the sake of the following comparison with observed Cepheids, we select the predicted fundamental pulsators with $\log P \geq 0.4$ and, by a regression through the synthetic populations we get the $P L$ relations listed in Table 4 (linear approximation) and Table 5 (quadratic approximation). One can see that the $l / H_{\mathrm{p}}$ variation from 1.5 to 1.7 produces minor effects on both the slope and zero-point, at fixed chemical composition. More importantly, present $P L$ relations confirm most of our previous results. In particular, we find that:

1. the pulsator distributions are better described by quadratic relations, mainly in the optical bands;

2. the slope and the intrinsic dispersion of the predicted periodmagnitude distribution at fixed metal content, decrease moving from optical to near-infrared bands, in agreement with well-known empirical results (e.g., Madore \& Freedman 1991);

3. decreasing the metal content, the linear $P L$ relations become steeper, depending on the filter wavelength: from $Z=0.02$ to 0.004 the slope of the $P-M_{B}, P-M_{V}$ and $P-M_{I}$ relations vary by $\sim 29 \%, 15 \%$ and $\sim 8 \%$, respectively, with no significant effects on the near-infrared relations;

4. regarding the zero-point of the $P L$ relations, at periods longer than $\sim 4-5$ days the metal-poor pulsators have

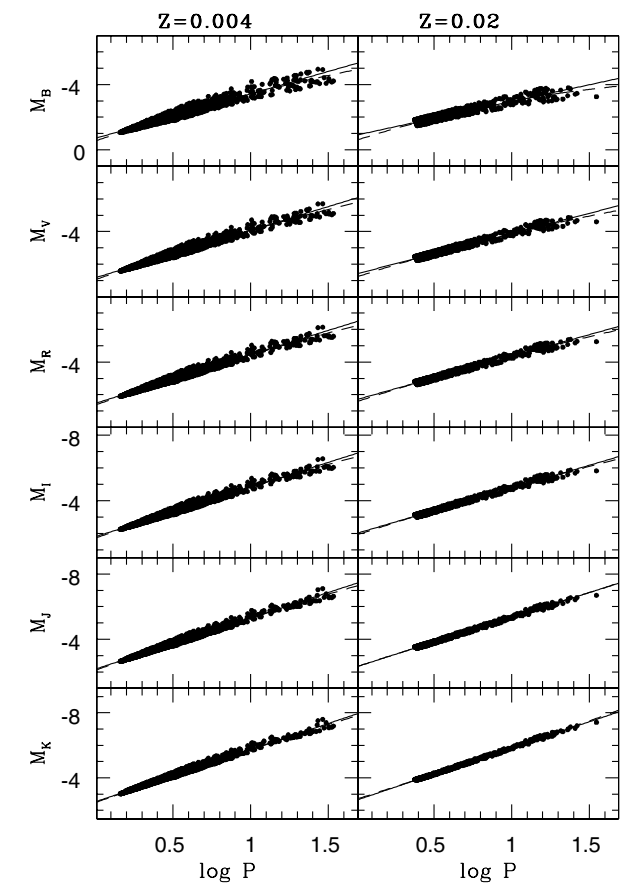

Fig. 7. Synthetic fundamental pulsators with $Z=0.004$ and $Y=0.251$ (left panels) and $Z=0.02, Y=0.289$ (right panel) adopting $l / H_{\mathrm{p}}=1.5$. The solid and the dashed lines refer to the linear and the quadratic fits, respectively.

brighter optical magnitudes than the metal-rich ones, again depending on the photometric band. Varying the metal content from $Z=0.02$ to 0.004 , the $B, V$ and $I$ magnitudes at $\log P=1$ become brighter by $\sim 0.47, \sim 0.18$ and $\sim 0.06 \mathrm{mag}$, whereas the $K$ magnitudes become fainter by $\sim 0.10 \mathrm{mag}$.

Finally, by comparing the new results at $l / H_{\mathrm{p}}=1.5$ with those presented in Paper V, we can estimate the effects of different methods to derive the synthetic $P L$ relations. As a whole, the new linear slopes appear somehow steeper $(\sim 6 \%)$ than our previous slopes, while the predicted magnitudes at $\log P=1$ agree within $\pm 0.1 \mathrm{mag}$.

\section{Theory versus observations}

To test the predictive potential of our pulsation scenario, we compare in the following the theoretical relations discussed in the previous sections with selected samples of Galactic and Magellanic Cloud Cepheids. 
Table 4. Synthetic linear $P L$ relations for fundamental pulsators with $\log P \geq 0.4$ in the form $M_{i}=\alpha+\beta \log P$.

\begin{tabular}{ccccccccc}
\hline \hline$M_{i}$ & $l / H_{\mathrm{p}}$ & $\alpha$ & $\beta$ & $\sigma$ & $l / H_{\mathrm{p}}$ & $\alpha$ & $\beta$ & $\sigma$ \\
\hline \multicolumn{7}{c}{$Z=0.004, Y=0.251$} \\
$M_{B}$ & 1.5 & $-0.78 \pm 0.02$ & $-2.64 \pm 0.03$ & 0.21 & 1.8 & - & - & - \\
$M_{V}$ & 1.5 & $-1.22 \pm 0.02$ & $-2.84 \pm 0.02$ & 0.16 & 1.8 & - & - & - \\
$M_{R}$ & 1.5 & $-1.52 \pm 0.01$ & $-2.92 \pm 0.02$ & 0.14 & 1.8 & - & - & - \\
$M_{I}$ & 1.5 & $-1.83 \pm 0.01$ & $-2.99 \pm 0.02$ & 0.13 & 1.8 & - & - & - \\
$M_{J}$ & 1.5 & $-2.20 \pm 0.01$ & $-3.09 \pm 0.02$ & 0.11 & 1.8 & - & - & - \\
$M_{K}$ & 1.5 & $-2.55 \pm 0.01$ & $-3.19 \pm 0.01$ & 0.10 & 1.8 & - & - & - \\
\hline \multicolumn{7}{c}{$Z=0.008, Y=0.251$} \\
$M_{B}$ & 1.5 & $-0.94 \pm 0.02$ & $-2.37 \pm 0.02$ & 0.29 & 1.8 & $-0.77 \pm 0.02$ & $-2.65 \pm 0.02$ & 0.23 \\
$M_{V}$ & 1.5 & $-1.35 \pm 0.02$ & $-2.73 \pm 0.02$ & 0.21 & 1.8 & $-1.22 \pm 0.02$ & $-2.94 \pm 0.02$ & 0.17 \\
$M_{R}$ & 1.5 & $-1.62 \pm 0.01$ & $-2.87 \pm 0.02$ & 0.18 & 1.8 & $-1.52 \pm 0.01$ & $-3.04 \pm 0.01$ & 0.14 \\
$M_{I}$ & 1.5 & $-1.91 \pm 0.01$ & $-3.00 \pm 0.01$ & 0.15 & 1.8 & $-1.82 \pm 0.01$ & $-3.15 \pm 0.01$ & 0.12 \\
$M_{J}$ & 1.5 & $-2.24 \pm 0.01$ & $-3.18 \pm 0.01$ & 0.11 & 1.8 & $-2.18 \pm 0.01$ & $-3.29 \pm 0.01$ & 0.09 \\
$M_{K}$ & 1.5 & $-2.54 \pm 0.01$ & $-3.35 \pm 0.01$ & 0.07 & 1.8 & $-2.51 \pm 0.01$ & $-3.41 \pm 0.01$ & 0.06 \\
\hline \multicolumn{7}{c}{$Z=0.02, Y=0.289$} & & \\
$M_{B}$ & 1.5 & $-0.90 \pm 0.01$ & $-2.05 \pm 0.02$ & 0.16 & 1.7 & $-0.90 \pm 0.02$ & $-2.14 \pm 0.03$ & 0.18 \\
$M_{V}$ & 1.5 & $-1.41 \pm 0.01$ & $-2.48 \pm 0.02$ & 0.11 & 1.7 & $-1.41 \pm 0.01$ & $-2.53 \pm 0.02$ & 0.13 \\
$M_{R}$ & 1.5 & $-1.70 \pm 0.01$ & $-2.65 \pm 0.01$ & 0.10 & 1.7 & $-1.70 \pm 0.01$ & $-2.69 \pm 0.02$ & 0.11 \\
$M_{I}$ & 1.5 & $-2.00 \pm 0.01$ & $-2.78 \pm 0.01$ & 0.08 & 1.7 & $-2.00 \pm 0.01$ & $-2.82 \pm 0.02$ & 0.09 \\
$M_{J}$ & 1.5 & $-2.32 \pm 0.01$ & $-3.03 \pm 0.01$ & 0.06 & 1.7 & $-2.33 \pm 0.01$ & $-3.04 \pm 0.01$ & 0.06 \\
$M_{K}$ & 1.5 & $-2.62 \pm 0.01$ & $-3.22 \pm 0.01$ & 0.04 & 1.7 & $-2.64 \pm 0.01$ & $-3.21 \pm 0.01$ & 0.04 \\
\hline
\end{tabular}

Table 5. Synthetic quadratic $P L$ relations for fundamental pulsators with $\log P \geq 0.4$ in the form $M_{i}=\alpha+\beta \log P+\gamma \log P^{2}$. Note that for each relation the rms dispersion is included in the error of the $\alpha$ coefficient.

\begin{tabular}{|c|c|c|c|c|c|c|c|c|}
\hline$M_{i}$ & $l / H_{\mathrm{p}}$ & $\alpha$ & $\beta$ & $\gamma$ & $l / H_{\mathrm{p}}$ & $\alpha$ & $\bar{\beta}$ & $\gamma$ \\
\hline \multicolumn{9}{|c|}{$Z=0.004, Y=0.251$} \\
\hline$M_{B}$ & 1.5 & $-0.41 \pm 0.10$ & $-3.64 \pm 0.15$ & $+0.60 \pm 0.09$ & 1.8 & \multicolumn{3}{|c|}{-} \\
\hline$M_{V}$ & 1.5 & $-0.95 \pm 0.15$ & $-3.59 \pm 0.12$ & $+0.46 \pm 0.07$ & 1.8 & - & \multicolumn{2}{|l|}{-} \\
\hline$M_{R}$ & 1.5 & $-1.27 \pm 0.13$ & $-3.58 \pm 0.11$ & $+0.40 \pm 0.06$ & 1.8 & - & \multicolumn{2}{|l|}{-} \\
\hline$M_{I}$ & 1.5 & $-1.62 \pm 0.11$ & $-3.58 \pm 0.10$ & $+0.35 \pm 0.06$ & 1.8 & - & \multicolumn{2}{|l|}{-} \\
\hline$M_{J}$ & 1.5 & $-2.03 \pm 0.08$ & $-3.57 \pm 0.08$ & $+0.29 \pm 0.05$ & 1.8 & - & \multicolumn{2}{|l|}{-} \\
\hline$M_{K}$ & 1.5 & $-2.41 \pm 0.06$ & $-3.57 \pm 0.08$ & $+0.23 \pm 0.05$ & 1.8 & - & \multicolumn{2}{|l|}{-} \\
\hline \multicolumn{9}{|c|}{$Z=0.008, Y=0.251$} \\
\hline$M_{B}$ & 1.5 & $-0.21 \pm 0.13$ & $-4.26 \pm 0.15$ & $+1.04 \pm 0.08$ & 1.8 & $+0.09 \pm 0.10$ & $-4.92 \pm 0.14$ & $+1.28 \pm 0.07$ \\
\hline$M_{V}$ & 1.5 & $-0.83 \pm 0.20$ & $-4.08 \pm 0.11$ & $+0.74 \pm 0.06$ & 1.8 & $-0.61 \pm 0.16$ & $-4.56 \pm 0.10$ & $+0.91 \pm 0.06$ \\
\hline$M_{R}$ & 1.5 & $-1.18 \pm 0.17$ & $-4.00 \pm 0.09$ & $+0.63 \pm 0.05$ & 1.8 & $-1.00 \pm 0.13$ & $-4.41 \pm 0.08$ & $+0.77 \pm 0.05$ \\
\hline$M_{I}$ & 1.5 & $-1.54 \pm 0.14$ & $-3.95 \pm 0.08$ & $+0.52 \pm 0.04$ & 1.8 & $-1.39 \pm 0.11$ & $-4.29 \pm 0.07$ & $+0.64 \pm 0.04$ \\
\hline$M_{J}$ & 1.5 & $-1.98 \pm 0.10$ & $-3.85 \pm 0.06$ & $+0.37 \pm 0.03$ & 1.8 & $-1.87 \pm 0.08$ & $-4.10 \pm 0.05$ & $+0.46 \pm 0.03$ \\
\hline$M_{K}$ & 1.5 & $-2.38 \pm 0.06$ & $-3.77 \pm 0.04$ & $+0.23 \pm 0.02$ & 1.8 & $-2.31 \pm 0.05$ & $-3.93 \pm 0.04$ & $+0.29 \pm 0.02$ \\
\hline \multicolumn{9}{|c|}{$Z=0.02, Y=0.289$} \\
\hline$M_{B}$ & 1.5 & $-0.55 \pm 0.08$ & $-3.09 \pm 0.14$ & $+0.66 \pm 0.09$ & 1.7 & $-0.69 \pm 0.10$ & $-2.79 \pm 0.18$ & $+0.45 \pm 0.12$ \\
\hline$M_{V}$ & 1.5 & $-1.19 \pm 0.12$ & $-3.12 \pm 0.10$ & $+0.41 \pm 0.06$ & 1.7 & $-1.30 \pm 0.16$ & $-2.87 \pm 0.13$ & $+0.23 \pm 0.09$ \\
\hline$M_{R}$ & 1.5 & $-1.54 \pm 0.10$ & $-3.13 \pm 0.09$ & $+0.31 \pm 0.05$ & 1.7 & $-1.64 \pm 0.13$ & $-2.90 \pm 0.11$ & $+0.14 \pm 0.08$ \\
\hline$M_{I}$ & 1.5 & $-1.87 \pm 0.09$ & $-3.15 \pm 0.07$ & $+0.23 \pm 0.05$ & 1.7 & $-1.96 \pm 0.11$ & $-2.94 \pm 0.09$ & $+0.08 \pm 0.06$ \\
\hline$M_{J}$ & 1.5 & $-2.29 \pm 0.06$ & $-3.10 \pm 0.05$ & $+0.04 \pm 0.03$ & 1.7 & $-2.36 \pm 0.07$ & $-2.94 \pm 0.06$ & $-0.07 \pm 0.04$ \\
\hline$M_{K}$ & 1.5 & $-2.67 \pm 0.04$ & $-3.09 \pm 0.04$ & $-0.09 \pm 0.02$ & 1.7 & $-2.71 \pm 0.04$ & $-2.98 \pm 0.04$ & $-0.16 \pm 0.03$ \\
\hline
\end{tabular}

\subsection{Galactic Cepheids with absolute trigonometric parallaxes}

The absolute VIK magnitudes determined by Benedict et al. (2007, hereafter B07) on the basis of HST trigonometric parallaxes yield the $P L$ relations $\left(M_{i}=\alpha+\beta \log P\right)$ reported in Table 6. Based on these absolute magnitudes and adopting $A_{V} / E(B-V)=3.3$ and $A_{V} / E(V-I)=2.52$, we derive the $P W$ relations listed in the same Table. Note that the $P-W B V$ relation is determined by using the $(B-V)$ and $E(B-V)$ values given by $\mathrm{B} 07$.

Bearing in mind that the $P L$ relations, mostly in the optical bands, depend on the finite width of the pulsation region and on the stellar distribution within the instability strip, the observed slope and zero-point provided by the B07 Cepheids appear comfortably consistent with the predicted values at $Z=0.02$ presented in Table 4. It is also worth noting that, compared with the results given in Table 11 for LMC $(Z=0.008)$ Cepheids, as determined by hundreds of variables observed by the OGLE team and Persson and coworkers (see later), the Galactic $P L$ relations in the $V I$ bands have a milder slope, in agreement with the predicted behavior. Regarding the $P W$ relations, which are less affected by the finite width of the instability strip, the Galactic $P-W V I$ and $P-W V K$ slopes differ by only $\sim 5 \%$ from the predicted values at constant metal content and $L / L_{\text {can }}$ ratio (Table 3), whereas the observed $P-W B V$ relation is statistically steeper than that predicted.

To investigate this discrepancy, we analyze the Cepheid apparent magnitudes of the B07 sample to show the capabilities of the predicted relations to obtain useful information on several stellar parameters, as summarized in Table 7 . Note that 
Table 6. Absolute $P L$ and $P W$ relations for fundamental Galactic Cepheids with measured distance. The near-infrared magnitudes are in the CIT photometric system.

\begin{tabular}{cccccc}
\hline \hline$M_{i}$ & $\alpha$ & $\beta$ & $W$ & $\alpha$ & $\beta$ \\
\hline \multicolumn{5}{c}{ B07 } \\
$M_{V}$ & -1.62 & -2.43 & $W B V$ & -2.03 & -4.52 \\
$M_{I}$ & -1.97 & -2.81 & $W V I$ & -2.47 & -3.43 \\
$M_{K}$ & -2.39 & -3.32 & $W V K$ & -2.45 & -3.43 \\
\multicolumn{7}{c}{$\mathrm{S} 04$} \\
$M_{V}$ & -0.95 & -3.08 & $W B V$ & -2.04 & -4.35 \\
$M_{I}$ & -1.49 & -3.30 & $W V I$ & -2.11 & -3.78 \\
$M_{J}$ & -1.77 & -3.53 & $W V K$ & -2.00 & -3.78 \\
$M_{K}$ & -2.02 & -3.67 & $W J K$ & -2.08 & -3.80 \\
\hline
\end{tabular}

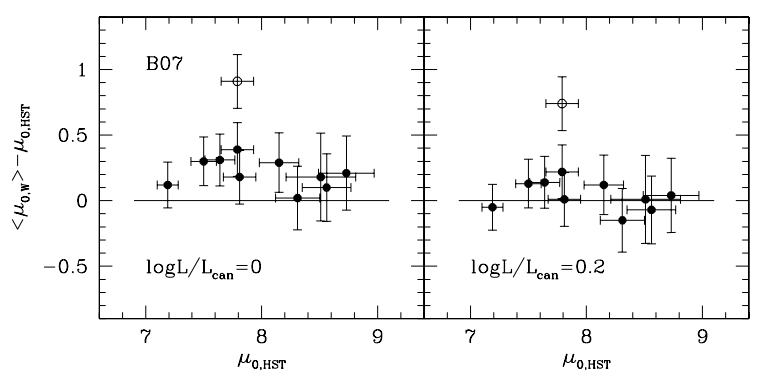

Fig. 8. Pulsation distance moduli of Galactic Cepheids versus $H S T$ based values under two assumptions of the $\log L / L_{\mathrm{can}}$ ratio. The open circle refers to FF Aql, if it were an overtone pulsator (see text).

the last line in this table deals with the case that FF Aql is an overtone pulsator (Antonello et al. 1990; Kienzle et al. 1999). Accordingly, the observed period has been increased by $\delta \log P=0.156$, as suggested by the pulsation theory (Alcock et al. 1995), in order to use our predicted fundamental relations.

As a first step, we adopt $\log L / L_{\text {can }}=0$ (canonical evolutionary frame) and the solar metallicity $Z_{\odot}=0.017$ to estimate the "pulsation" distance moduli $\mu_{0}$ from the predicted $P W_{\mathrm{e}}$ relations and the observed $W B V, W V I$ and $W V K$ quantities. Note that our models adopt the Bessell \& Brett (1988) near-infrared system and for this reason the $K(\mathrm{CIT})$ magnitudes have been increased by 0.02 mag according to Carpenter (2001). As shown in the Cols. 4 to 6 in Table 7, the distances given by the $W B V$ functions are generally longer than the $W V I$ and $W V K$-based values, with the only exception of $1 \mathrm{Car}$ which shows an opposite behaviour. Then, by exploiting the metallicity dependence of the various $P W_{\mathrm{e}}$ relations (see Table 3 ), we demand that $W B V$, $W V I$ and $W V K$ provide the same distance and we eventually derive the $Z$ and $\left\langle\mu_{0, W}\right\rangle$ values listed in Cols. 7 and 8 , respectively, while the following two columns give the results inferred by repeating the procedure with $\log L / L_{\text {can }}=0.2$.

As a whole, we get metal abundances which are quite consistent with a solar-like value and pulsation distances in statistical agreement with the parallax-based values, closer for the non-canonical value $\log L / L_{\text {can }}=0.2$ (Fig. 8). However, since $\delta\left\langle\mu_{0, W}\right\rangle=-0.85 \delta \log L / L_{\text {can }}$, we take the $H S T$-based distance in Col. 3 at face value to derive the final $\log L / L_{\text {can }}$ and $Z$ values of each individual variable, as listed in the last two columns in the Table. For all the variables we find positive $\log L / L_{\text {can }}$ ratios which suggest an average value of about 0.25 or a fair period dependence as $\log L / L_{\text {can }}=0.41-0.19 \log P$. Regarding FF Aql, if it were an overtone pulsator, it would have an exceedingly large $\log L / L_{\text {can }}$ ratio to fit the $H S T$-based distance, leading us to conclude that this variable is a fundamental pulsator.

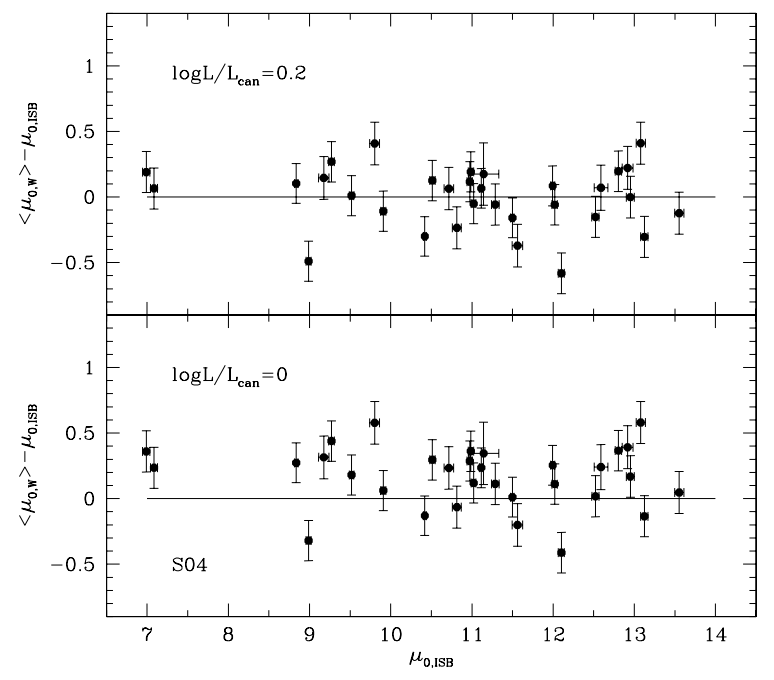

Fig. 9. Pulsation distance moduli of Galactic Cepheids versus ISBbased values under two assumptions of the $\log L / L_{\text {can }}$ ratio.

All variables yield $\log Z=-2.0 \pm 0.1$, with the exception of 1 Car which seems to be significantly more metal-rich. Indeed, the high-resolution spectroscopic measurements by Romaniello et al. (2005) and Lemasle et al. (2007) for $\beta$ Dor $([\mathrm{Fe} / \mathrm{H}]=$ $-0.14), \zeta \mathrm{Gem}([\mathrm{Fe} / \mathrm{H}]=-0.19)$ and $1 \mathrm{Car}([\mathrm{Fe} / \mathrm{H}]=+0.10)$ seem to support the metal overabundance of $1 \mathrm{Car}$ with respect to the other variables. It is of interest to note that the exclusion of 1 Car would actually lead to mildly steeper $P-M_{V}$ and $P-M_{I}$ relations, i.e. $M_{V}=-1.43-2.67 \log P$ and $M_{I}=-1.82-3.01 \log P$, but is still shallower than the LMC counterparts (Table 11). Furthermore, we note that, with respect to these new relations at $[\mathrm{Fe} / \mathrm{H}] \sim-2.0,1 \mathrm{Car}([\mathrm{Fe} / \mathrm{H}]=+0.10)$ turns out to be fainter by $\delta M_{V}=0.22 \mathrm{mag}$ and $\delta M_{I}=0.18 \mathrm{mag}$, in agreement with the predicted metallicity effect.

Finally, note that using the HST-based distance in Col. 3 and the metal content in Col. 12 together with the predicted massdependent $P W$ relations in Table 2 we estimate that the mass of W Sgr is $6.0 \pm 1.5 M_{\odot}$, in close agreement with the value $6.5 \pm 2 M_{\odot}$ listed by B07. Regarding FF Aql, we find 3.5 $\pm 1.5 M_{\odot}$ (fundamental pulsator) and $2.3 \pm 1.5 M_{\odot}$ (first overtone) which, compared with the B07 mass $4.5 \pm 1 M_{\odot}$, should again support that this variable pulsates in the fundamental mode. The comparison between the evolutionary and the pulsation mass of Galactic and LMC Cepheids will be discussed in a forthcoming paper.

\subsection{Galactic Cepheids with ISB distances}

The absolute magnitudes BVIJK determined by S04 using the Infrared Surface Brightness (ISB) technique provide the absolute $P L$ and $P W$ relations reported in Table 6 . These relations are significantly steeper in comparison with the predicted linear relations at solar metal content, as well as with respect to the B07 and the LMC results listed in Tables 6 and 11, respectively.

By repeating the procedure adopted for the B07 sample, the S04 variables yield the results listed in Table 8, while Fig. 9 shows that no statistical agreement between pulsation and ISBbased distances is found with $\log L / L_{\mathrm{can}}=0$ nor 0.2 . As a fact, based on the $\left\langle\mu_{0, W}\right\rangle-\log L / L_{\text {can }}$ relation, we use the ISB-based distances to determine the ratios listed in Col. 11 in Table 8 and we draw the attention to the resulting quite large spread as well as to some unrealistic negative values towards the longer periods. Moreover, a straight regression through the results yields 
Table 7. Galactic Cepheids with distance based on the HST trigonometric parallax measured by B07. From left to right, the columns give: name (1), period (2), absolute distance modulus (mag) based on the HST trigonometric parallax, with the average associated error (3), absolute distance moduli determined by the predicted evolutionary $P$-WBV (4), $P$-WVI (5) and $P$-WVK (6) relations adopting $Z=0.017$ and $\log L / L_{\mathrm{can}}=0$. By imposing that the three $P W$ relations give the same result, we get the metal content and pulsation distance modulus at $\log L / L_{\mathrm{can}}=0(\mathrm{Cols}$. 7 and 8 , respectively) and $\log L / L_{\text {can }}=0.2$ (Cols. 9 and 10, respectively). The comparison with the $H S T$-based distance modulus yields the final $\log L / L_{\text {can }}$ value and metal content listed in the last two columns.

\begin{tabular}{|c|c|c|c|c|c|c|c|c|c|c|c|}
\hline Name & $\log P$ & $\begin{array}{c}\mu_{0, H S T} \\
\quad( \pm)\end{array}$ & $\begin{array}{c}\mu_{0, W B V} \\
\pm 0.06\end{array}$ & $\begin{array}{c}\mu_{0, W V I} \\
\pm 0.12\end{array}$ & $\begin{array}{c}\mu_{0, W V K} \\
\pm 0.10\end{array}$ & $\begin{array}{l}\log Z \\
\pm 0.15\end{array}$ & $\begin{array}{l}\left\langle\mu_{0, W}\right\rangle \\
\pm 0.15\end{array}$ & $\begin{array}{l}\log Z \\
\pm 0.15\end{array}$ & $\begin{array}{c}\left\langle\mu_{0, W}\right\rangle \\
\pm 0.15\end{array}$ & $\begin{array}{c}\log L / L_{\text {can }} \\
( \pm)\end{array}$ & $\begin{array}{l}\log Z \\
\pm 0.15\end{array}$ \\
\hline 1 & 2 & 3 & 4 & 5 & 6 & 7 & 8 & 9 & 10 & 11 & 12 \\
\hline RTAur & 0.572 & $8.15(0.17)$ & 8.62 & 8.43 & 8.40 & -2.02 & 8.44 & -2.06 & 8.27 & $+0.34(0.20)$ & -2.09 \\
\hline TVul & 0.647 & $8.73(0.24)$ & 9.06 & 8.93 & 8.91 & -1.95 & 8.94 & -1.98 & 8.77 & $+0.24(0.28)$ & -1.99 \\
\hline FFAql & 0.650 & $7.79(0.14)$ & 8.30 & 8.16 & 8.17 & -1.94 & 8.18 & -1.98 & 8.01 & $+0.46(0.16)$ & -2.02 \\
\hline$\delta$ Cep & 0.730 & $7.19(0.09)$ & 7.52 & 7.27 & 7.30 & -2.06 & 7.31 & -2.09 & 7.14 & $+0.14(0.11)$ & -2.08 \\
\hline YSgr & 0.761 & $8.51(0.30)$ & 8.77 & 8.73 & 8.63 & -1.88 & 8.69 & -1.91 & 8.52 & $+0.21(0.35)$ & -1.92 \\
\hline XSgr & 0.846 & $7.64(0.13)$ & 8.29 & 7.90 & 7.90 & -2.25 & 7.95 & -2.29 & 7.78 & $+0.36(0.15)$ & -2.32 \\
\hline WSgr & 0.881 & $8.31(0.19)$ & 8.51 & 8.36 & 8.27 & -2.02 & 8.33 & -2.05 & 8.16 & $+0.03(0.22)$ & -2.02 \\
\hline$\beta$ Dor & 0.993 & $7.50(0.11)$ & 7.83 & 7.80 & 7.80 & -1.80 & 7.80 & -1.84 & 7.63 & $+0.36(0.13)$ & -1.86 \\
\hline$\zeta \mathrm{Gem}$ & 1.007 & $7.81(0.14)$ & 8.07 & 7.96 & 8.00 & -1.88 & 7.99 & -1.92 & 7.82 & $+0.21(0.16)$ & -1.92 \\
\hline 1Car & 1.551 & $8.56(0.21)$ & 8.32 & 8.70 & 8.71 & -1.30 & 8.66 & -1.33 & 8.49 & $+0.12(0.26)$ & -1.31 \\
\hline FFAql(FO) & 0.806 & $7.79(0.14)$ & 8.90 & 8.67 & 8.69 & -2.04 & 8.70 & -2.08 & 8.53 & $+1.08(0.16)$ & -2.24 \\
\hline
\end{tabular}

$\log L / L_{\text {can }}=0.85-0.55 \log P$, which is significantly different from the B07 Cepheid result.

Note that G05 have already shown that the ISB distances to 13 LMC Cepheids, even corrected for the tilt of the LMC bar, show a puzzling dependence on the period, with the shortest period variables giving $\mu_{0}(\mathrm{LMC}) \sim 18.3 \mathrm{mag}$ and the longest period ones $\sim 18.6 \mathrm{mag}$ ( see values listed in Col. 3 in Table 12), and yield $P L$ relations significantly steeper (see Col. 3 in Table 9) than those determined by the OGLE and P04's very large samples. On this basis, having identified that the adopted $\mathrm{p}$-factor which is used to convert radial into pulsation velocities is most likely responsible for this behavior, they suggest a correction to the former ISB distances as given by

$$
\Delta \mu_{0}\left(I S B_{\text {new }}-I S B_{\text {old }}\right)=0.29-0.18 \log P
$$

with an LMC distance modulus $\mu_{0}(L M C)=18.56$ mag. Using this correction to re-determine the distances to the LMC and S04 Galactic Cepheids, they eventually attain agreement with the OGLE and P04 PL relations and no significant difference between the slopes of the various relations between Galactic and LMC variables (see last two columns in Table 9). However, we note that the slope variation $(\Delta \beta=+0.18)$ produced by the G05 correction also results in the revised $P L$ and $P W$ relations of the S04 Cepheids being steeper in comparison with the B07 results. Furthermore, using Eq. (3) to correct the ISB distance moduli of the S04 Cepheids we find $\log L / L_{\text {can }}=0.50-0.32 \log P$, which is still different from the result inferred by the B07 Cepheids.

In fact, the comparison between the $H S T$ and ISB distances for $\delta$ Cep $(\log P=0.730)$ and 1 Car $(\log P=1.551)$, the only two common variables, gives $\Delta \mu_{0}\left(H S T-I S B_{\text {old }}\right)=0.10 \pm 0.10 \mathrm{mag}$ and $-0.43 \pm 0.21 \mathrm{mag}$, respectively, while the revised ISB distances give $\Delta \mu_{0}\left(H S T-I S B_{\text {rev }}\right)=-0.06 \pm 0.10 \mathrm{mag}$ for the short-period Cepheid and $-0.44 \pm 0.21 \mathrm{mag}$ for the long-period, suggesting a more significant period-dependent correction to the ISB distances. On the other hand, it seems reasonable to assume that the Galactic Cepheids in the two samples follow similar relations and/or evolutionary prescriptions. On this basis, we show in Fig. 10 that assuming for the S04 Cepheids the absolute $P$ $W V I$ and $P-W V K$ relations provided by the B07 variables the correction is

$\Delta \mu_{0}\left(p u l s-I S B_{\text {old }}\right)=0.41-0.35 \log P$,

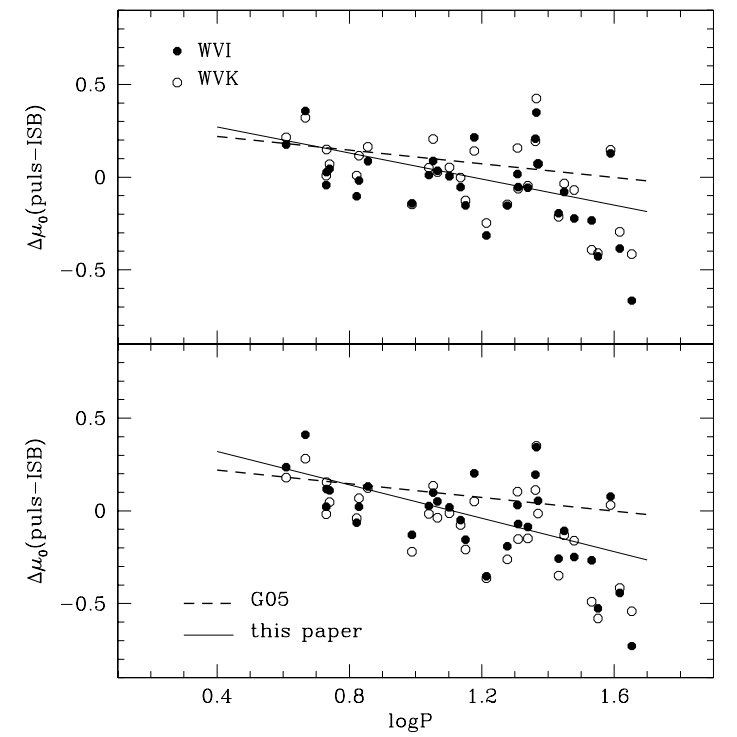

Fig. 10. (Upper panel) Corrections to ISB-based distances to S04 Cepheids using the $P-W V I$ and $P-W V K$ relations provided by the B07 variables. (Lower panel) As for the upper panel, but adopting $\log L / L_{\text {can }}=0.25$, which is the average value of the B07 Cepheids. The solid line is the linear regression through the points, while the dashed line is the correction suggested by G05.

while adopting $\log L / L_{\text {can }}=0.25$, which is the average value of the B07 Cepheids, one has

$\Delta \mu_{0}\left(\right.$ puls $\left.-I S B_{\text {old }}\right)=0.50-0.45 \log P$.

It goes without saying that these two $H S T$-based corrections would lead the $P L$ and $P W$ relations of the S04 Cepheids to agree with the B07 results.

\subsection{Galactic Cepheids with measured $[\mathrm{Fe} / \mathrm{H}]$}

The evolutionary $P W_{\mathrm{e}}$ relations presented in Table 3 give the distance to Cepheids with known metal content, once the $\log L / L_{\text {can }}$ ratio is adopted. On the other hand, the opposite metallicity dependence of $W B V$ with respect to $W V I$ and $W V K$ provides a direct way to estimate the metal content of Cepheids with $B V I$ 
Table 8. As in Table 7, but for the Galactic Cepheids with distance derived by S04 using the Infrared Surface Brightness (ISB) technique. From left to right, the columns give: name (1), period (2), absolute distance modulus (mag) based on the ISB method (3), absolute distance moduli determined by the predicted evolutionary $P-W B V(4), P$-WVI (5) and $P-W V K$ (6) relations adopting $Z=0.017$ and $\log L / L_{\mathrm{can}}=0$. By imposing that the three $P W$ relations give the same result, we get the metal content and pulsation distance modulus at $\log L / L_{\text {can }}=0$ (Cols. 7 and 8 , respectively) and $\log L / L_{\text {can }}=0.2$ (Cols. 9 and 10, respectively). The comparison with the ISB-based distance modulus yields the final $\log L / L_{\text {can }}$ value and metal content listed in the last two columns.

\begin{tabular}{|c|c|c|c|c|c|c|c|c|c|c|c|}
\hline Name & $\log P$ & $\begin{array}{c}\mu_{0, I S B} B \\
( \pm)\end{array}$ & $\begin{array}{c}\mu_{0, W B V} \\
\pm 0.06\end{array}$ & $\begin{array}{c}\mu_{0, W V I} \\
\pm 0.12\end{array}$ & $\begin{array}{c}\mu_{0, W V K} \\
\pm 0.10\end{array}$ & $\begin{array}{l}\log Z \\
\pm 0.15\end{array}$ & $\begin{array}{l}\left\langle\mu_{0, W}\right\rangle \\
\pm 0.15\end{array}$ & $\begin{array}{l}\log Z \\
\pm 0.15\end{array}$ & $\begin{array}{l}\left\langle\mu_{0, W}\right\rangle \\
\pm 0.15\end{array}$ & $\begin{array}{c}\log L / L_{\mathrm{can}} \\
( \pm)\end{array}$ & $\begin{array}{l}\log Z \\
\pm 0.15\end{array}$ \\
\hline 1 & 2 & 3 & 4 & 5 & 6 & 7 & 8 & 9 & 10 & 11 & 12 \\
\hline BF Oph & 0.609 & $9.27(0.03)$ & 9.75 & 9.71 & 9.68 & -1.84 & 9.70 & -1.88 & 9.53 & $+0.50(0.15)$ & -1.93 \\
\hline T Vel & 0.667 & $9.80(0.06)$ & 10.47 & 10.41 & 10.31 & -1.90 & 10.37 & -1.94 & 10.20 & $+0.67(0.16)$ & -2.03 \\
\hline$\delta$ Cер & 0.730 & $7.09(0.04)$ & 7.53 & 7.29 & 7.27 & -2.08 & 7.31 & -2.12 & 7.14 & $+0.26(0.16)$ & -2.13 \\
\hline CV Mon & 0.731 & $10.99(0.03)$ & 11.77 & 11.26 & 11.32 & -2.37 & 11.34 & -2.41 & 11.17 & $+0.41(0.15)$ & -2.46 \\
\hline V Cen & 0.740 & $9.18(0.06)$ & 9.73 & 9.47 & 9.43 & -2.12 & 9.48 & -2.16 & 9.30 & $+0.35(0.16)$ & -2.19 \\
\hline BB Sgr & 0.822 & $9.52(0.03)$ & 9.77 & 9.65 & 9.70 & -1.88 & 9.69 & -1.92 & 9.52 & $+0.20(0.15)$ & -1.92 \\
\hline U Sgr & 0.829 & $8.84(0.02)$ & 9.18 & 9.05 & 9.13 & -1.89 & 9.10 & -1.93 & 8.93 & $+0.31(0.15)$ & -1.95 \\
\hline$\eta$ Aql & 0.856 & $6.99(0.05)$ & 7.52 & 7.31 & 7.32 & -2.03 & 7.34 & -2.07 & 7.17 & $+0.41(0.16)$ & -2.11 \\
\hline S Nor & 0.989 & $9.91(0.03)$ & 10.03 & 9.98 & 9.92 & -1.88 & 9.96 & -1.91 & 9.79 & $+0.06(0.15)$ & -1.89 \\
\hline XX Cen & 1.040 & $11.11(0.02)$ & 11.49 & 11.33 & 11.32 & -1.98 & 11.34 & -2.01 & 11.17 & $+0.27(0.15)$ & -2.03 \\
\hline V340 Nor & 1.053 & $11.15(0.19)$ & 11.56 & 11.44 & 11.50 & -1.88 & 11.48 & -1.92 & 11.31 & $+0.39(0.24)$ & -1.96 \\
\hline UU Mus & 1.066 & $12.59(0.08)$ & 13.03 & 12.83 & 12.77 & -2.06 & 12.82 & -2.10 & 12.65 & $+0.27(0.17)$ & -2.11 \\
\hline U Nor & 1.102 & $10.72(0.06)$ & 11.14 & 10.92 & 10.91 & -2.05 & 10.94 & -2.09 & 10.77 & $+0.26(0.16)$ & -2.10 \\
\hline BN Pup & 1.136 & $12.95(0.05)$ & 13.26 & 13.09 & 13.09 & -1.98 & 13.11 & -2.01 & 12.94 & $+0.18(0.16)$ & -2.01 \\
\hline LS Pup & 1.151 & $13.55(0.06)$ & 13.71 & 13.59 & 13.57 & -1.93 & 13.59 & -1.97 & 13.42 & $+0.05(0.16)$ & -1.94 \\
\hline VW Cen & 1.177 & $12.80(0.04)$ & 13.27 & 13.21 & 13.09 & -1.93 & 13.16 & -1.96 & 12.99 & $+0.42(0.16)$ & -2.01 \\
\hline X Cyg & 1.214 & $10.42(0.02)$ & 10.15 & 10.29 & 10.31 & -1.58 & 10.28 & -1.62 & 10.11 & $-0.16(0.15)$ & -1.55 \\
\hline VY Car & 1.277 & $11.50(0.02)$ & 11.45 & 11.52 & 11.48 & -1.71 & 11.50 & -1.74 & 11.33 & $-0.01(0.15)$ & -1.71 \\
\hline RY Sco & 1.308 & $10.51(0.03)$ & 11.20 & 10.70 & 10.80 & -2.34 & 10.80 & -2.38 & 10.63 & $+0.33(0.15)$ & -2.41 \\
\hline RZ Vel & 1.310 & $11.02(0.03)$ & 11.32 & 11.14 & 11.09 & -2.03 & 11.13 & -2.07 & 10.96 & $+0.13(0.15)$ & -2.06 \\
\hline WZ Sgr & 1.339 & $11.29(0.05)$ & 11.49 & 11.40 & 11.37 & -1.91 & 11.39 & -1.94 & 11.22 & $+0.13(0.16)$ & -1.93 \\
\hline WZ Car & 1.362 & $12.92(0.07)$ & 13.59 & 13.29 & 13.23 & -2.19 & 13.30 & -2.22 & 13.13 & $+0.44(0.17)$ & -2.27 \\
\hline VZ Pup & 1.365 & $13.08(0.06)$ & 13.97 & 13.59 & 13.62 & -2.22 & 13.65 & -2.26 & 13.47 & $+0.65(0.16)$ & -2.35 \\
\hline SW Vel & 1.370 & $12.00(0.03)$ & 12.49 & 12.23 & 12.19 & -2.13 & 12.24 & -2.17 & 12.07 & $+0.28(0.15)$ & -2.18 \\
\hline T Mon & 1.432 & $10.82(0.06)$ & 10.67 & 10.78 & 10.72 & -1.67 & 10.74 & -1.71 & 10.57 & $-0.09(0.16)$ & -1.65 \\
\hline RY Vel & 1.449 & $12.02(0.03)$ & 12.34 & 12.09 & 12.10 & -2.08 & 12.12 & -2.12 & 11.95 & $+0.11(0.15)$ & -2.10 \\
\hline AQ Pup & 1.479 & $12.52(0.05)$ & 12.76 & 12.45 & 12.56 & -2.09 & 12.53 & -2.13 & 12.36 & $+0.01(0.16)$ & -2.09 \\
\hline KN Cen & 1.532 & $13.12(0.05)$ & 13.34 & 13.03 & 12.84 & -2.28 & 12.98 & -2.32 & 12.81 & $-0.17(0.16)$ & -2.24 \\
\hline $1 \mathrm{Car}$ & 1.551 & $8.99(0.03)$ & 8.39 & 8.70 & 8.69 & -1.39 & 8.66 & -1.42 & 8.49 & $-0.39(0.15)$ & -1.32 \\
\hline U Car & 1.589 & $10.97(0.03)$ & 11.44 & 11.24 & 11.22 & -2.03 & 11.25 & -2.07 & 11.08 & $+0.32(0.15)$ & -2.09 \\
\hline RS Pup & 1.617 & $11.56(0.06)$ & 11.48 & 11.31 & 11.36 & -1.96 & 11.35 & -1.99 & 11.18 & $-0.25(0.16)$ & -1.91 \\
\hline SV Vul & 1.653 & $12.10(0.04)$ & 11.73 & 11.56 & 11.78 & -1.85 & 11.68 & -1.88 & 11.51 & $-0.50(0.16)$ & -1.75 \\
\hline
\end{tabular}

Table 9. Slopes of the $P L$ relations from canonical and revised ISB distances to Galactic (MW) and LMC Cepheids, adopting the correction suggested by G05.

\begin{tabular}{ccccc}
\hline \hline$M_{i}$ & $\begin{array}{c}\text { MW } \\
\text { (can) }\end{array}$ & $\begin{array}{c}\text { LMC } \\
\text { (can) }\end{array}$ & $\begin{array}{c}\text { MW } \\
\text { (rev) }\end{array}$ & $\begin{array}{c}\text { LMC } \\
\text { (rev) }\end{array}$ \\
\hline$M_{V}$ & -3.08 & -3.05 & -2.90 & -2.87 \\
$M_{I}$ & -3.30 & -3.29 & -3.13 & -3.11 \\
$M_{J}$ & -3.53 & -3.48 & -3.33 & -3.29 \\
$M_{K}$ & -3.67 & -3.54 & -3.46 & -3.36 \\
\hline
\end{tabular}

or $B V K$ magnitudes, independently of distance and reddening and scarcely influenced by the $L / L_{\text {can }}$ ratio.

To test the reliability of the method, we used the observed BVI magnitudes compiled by Berdnikov et al. (2000) for fundamental pulsators together with the extensive $[\mathrm{Fe} / \mathrm{H}]$ measurements by Andrievsky and coworkers (Andrievsky 2002a-c, 2004; Luck et al. 2003). The pulsation $\log Z$ and $\mu_{0}$ values at $\log L / L_{\text {can }}=0$, as derived by the observed $W B V$ and $W V I$ quantities together with the predicted relations given in Table 3, are listed in Cols. 8 and 9, respectively, in Table 10, while the last two columns give the resulting absolute $M_{V}$ and $M_{I}$ magnitudes adopting the $E(B-V)$ reddening (Fernie et al. 1995) given in Col. 3 in the table.

Figure 11 shows the comparison between the measured $[\mathrm{Fe} / \mathrm{H}]_{A}$ parameter and the pulsation metal content $\log Z$ at $\log L / L_{\text {can }}=0$. We find $\log Z=[\mathrm{Fe} / \mathrm{H}]_{A}-1.90 \pm 0.15$ which is somehow different from the canonical relation $\log Z=[\mathrm{Fe} / \mathrm{H}]-$ 1.77 (solid line) with $Z_{\odot}=0.017$. However, given the current discrepancy (Groenewegen et al. 2004) between abundance determinations by different authors, as shown in Fig. 12, the pulsation method seems to be sufficiently adequate to provide the Cepheid metal content with an uncertainty almost comparable with that of spectroscopic studies.

Using the pulsation distances in Col. 9 and the reddening in Col. 3, we determine the Cepheid absolute magnitudes $M_{V}$ and $M_{I}$ listed in the last two columns in Table 10. As shown in Fig. 13, the resulting $P-M_{V}$ and $P-M_{I}$ relations of these Galactic Cepheids with $[\mathrm{Fe} / \mathrm{H}]=-0.04 \pm 0.16$ are well defined and the linear regression through the points (solid line) is

$$
M_{V}=-1.74( \pm 0.15)-2.60( \pm 0.17) \log P
$$

and

$M_{I}=-2.18( \pm 0.09)-2.87( \pm 0.10) \log P$ 
Table 10. Fundamental Galactic Cepheids with $[\mathrm{Fe} / \mathrm{H}]$ measurements by Andrievsky and collaborators. From left to right, the columns give: name (1), period (2), reddening $E(B-V)(3), B V I$ magnitudes [(4)-(6)] and measured $[\mathrm{Fe} / \mathrm{H}]_{A}$ value (7). The pulsation metal content log $Z$ and distance modulus $\mu_{0}$ at $\log L / L_{\text {can }}=0$, as inferred by the observed $W B V$ and $W V I$ functions, are listed in Cols. 8 and 9 , respectively, while the last two columns give the resulting absolute magnitudes $M_{V}$ and $M_{I}$.

\begin{tabular}{|c|c|c|c|c|c|c|c|c|c|c|}
\hline Name & $\log P$ & $E(B-V)$ & $\bar{B}$ & $V$ & $I$ & $\begin{array}{r}\mathrm{Fe} / \mathrm{H}]_{A} \\
\pm 0.10\end{array}$ & $\begin{array}{r}\log Z \\
\pm 0.15\end{array}$ & $\begin{array}{r}\mu_{0} \\
\pm 0.15\end{array}$ & $\begin{array}{r}M_{V} \\
\pm 0.15\end{array}$ & $\begin{array}{r}M_{I} \\
\pm 0.15\end{array}$ \\
\hline 1 & 2 & 3 & 4 & 5 & 6 & 7 & 8 & 9 & 10 & 11 \\
\hline V504 Mon & 0.443 & 0.57 & 12.82 & 11.81 & 10.54 & -0.31 & -2.06 & 12.91 & -2.96 & -3.49 \\
\hline FI Mon & 0.517 & 0.54 & 14.07 & 12.95 & 11.57 & -0.18 & -2.01 & 14.01 & -2.84 & -3.51 \\
\hline V335 Aur & 0.533 & 0.66 & 13.62 & 12.46 & 11.04 & -0.27 & -1.97 & 13.47 & -3.18 & -3.74 \\
\hline RT Aur & 0.571 & 0.05 & 6.04 & 5.45 & 4.81 & +0.06 & -1.87 & 8.55 & -3.27 & -3.84 \\
\hline SU Cyg & 0.585 & 0.10 & 7.43 & 6.86 & 6.20 & -0.01 & -2.06 & 9.95 & -3.40 & -3.94 \\
\hline CS Ori & 0.590 & 0.41 & 12.34 & 11.39 & 10.26 & -0.26 & -1.95 & 13.32 & -3.28 & -3.87 \\
\hline AE Tau & 0.591 & 0.60 & 12.83 & 11.70 & 10.38 & -0.19 & -1.81 & 13.13 & -3.42 & -3.95 \\
\hline AA Mon & 0.595 & 0.83 & 14.07 & 12.74 & 11.09 & -0.21 & -2.08 & 13.36 & -3.37 & -3.94 \\
\hline EK Mon & 0.597 & 0.58 & 12.28 & 11.07 & 9.61 & -0.10 & -1.93 & 12.19 & -3.05 & -3.74 \\
\hline ST Tau & 0.606 & 0.36 & 9.07 & 8.22 & 7.14 & -0.05 & -2.21 & 10.35 & -3.31 & -3.92 \\
\hline V495 Mon & 0.612 & 0.64 & 13.69 & 12.44 & 10.97 & -0.26 & -1.80 & 13.57 & -3.24 & -3.87 \\
\hline V508 Mon & 0.616 & 0.32 & 11.39 & 10.50 & 9.46 & -0.25 & -1.97 & 12.73 & -3.30 & -3.92 \\
\hline VW Pup & 0.632 & 0.51 & 12.50 & 11.38 & 10.08 & -0.19 & -1.84 & 13.00 & -3.32 & -3.95 \\
\hline Y Lac & 0.636 & 0.22 & 9.88 & 9.15 & 8.30 & -0.09 & -2.00 & 11.93 & -3.50 & -4.07 \\
\hline T Vul & 0.647 & 0.06 & 6.40 & 5.75 & 5.08 & +0.01 & -1.83 & 9.00 & -3.45 & -4.05 \\
\hline FF Aql & 0.650 & 0.22 & 6.13 & 5.37 & 4.51 & +0.02 & -1.97 & 8.16 & -3.53 & -4.10 \\
\hline FG Mon & 0.653 & 0.68 & 14.44 & 13.24 & 11.74 & -0.20 & -2.10 & 14.47 & -3.48 & -4.08 \\
\hline $\mathrm{T}$ Vel & 0.667 & 0.28 & 8.97 & 8.04 & 6.96 & -0.02 & -1.90 & 10.34 & -3.24 & -3.94 \\
\hline WW Mon & 0.669 & 0.64 & 13.65 & 12.50 & 11.14 & -0.29 & -1.95 & 14.09 & -3.68 & -4.22 \\
\hline RY CMa & 0.670 & 0.25 & 8.95 & 8.11 & 7.13 & +0.02 & -1.94 & 10.69 & -3.40 & -4.05 \\
\hline CU Mon & 0.673 & 0.79 & 15.02 & 13.61 & 11.90 & -0.26 & -1.94 & 14.36 & -3.35 & -4.02 \\
\hline EE Mon & 0.682 & 0.49 & 14.03 & 13.02 & 11.70 & -0.51 & -2.35 & 14.81 & -3.41 & -4.08 \\
\hline CF Cas & 0.688 & 0.57 & 12.34 & 11.14 & 9.75 & -0.01 & -1.78 & 12.74 & -3.47 & -4.11 \\
\hline BG Lac & 0.727 & 0.34 & 9.85 & 8.89 & 7.81 & -0.01 & -1.83 & 11.40 & -3.62 & -4.25 \\
\hline$\delta$ Cep & 0.730 & 0.09 & 4.62 & 3.96 & 3.20 & +0.06 & -2.06 & 7.30 & -3.65 & -4.28 \\
\hline CV Mon & 0.731 & 0.71 & 11.61 & 10.30 & 8.65 & -0.03 & -2.26 & 11.39 & -3.44 & -4.16 \\
\hline XX Mon & 0.737 & 0.60 & 13.10 & 11.92 & 10.50 & -0.10 & -1.98 & 13.61 & -3.66 & -4.30 \\
\hline V Cen & 0.740 & 0.29 & 7.69 & 6.82 & 5.81 & +0.04 & -2.00 & 9.53 & -3.67 & -4.30 \\
\hline WW Pup & 0.742 & 0.40 & 11.47 & 10.61 & 9.51 & -0.18 & -2.39 & 13.13 & -3.83 & -4.42 \\
\hline RZ Gem & 0.743 & 0.57 & 11.06 & 10.02 & 8.75 & -0.12 & -2.16 & 12.10 & -3.96 & -4.49 \\
\hline Y Sgr & 0.761 & 0.21 & 6.60 & 5.74 & 4.78 & +0.06 & -1.94 & 8.65 & -3.58 & -4.28 \\
\hline FM Aql & 0.786 & 0.65 & 9.58 & 8.28 & 6.78 & +0.08 & -1.76 & 9.92 & -3.78 & -4.43 \\
\hline X Vul & 0.801 & 0.85 & 10.24 & 8.84 & 7.20 & +0.08 & -1.88 & 10.16 & -4.11 & -4.65 \\
\hline U Sgr & 0.829 & 0.40 & 7.79 & 6.70 & 5.45 & +0.04 & -1.87 & 9.11 & -3.74 & -4.46 \\
\hline TW Mon & 0.851 & 0.70 & 13.91 & 12.57 & 10.95 & -0.24 & -2.01 & 14.14 & -3.87 & -4.57 \\
\hline$\eta \mathrm{Aql}$ & 0.856 & 0.15 & 4.69 & 3.90 & 3.03 & +0.05 & -1.95 & 7.37 & -3.97 & -4.63 \\
\hline V510 Mon & 0.864 & 0.84 & 14.13 & 12.65 & 10.86 & -0.19 & -2.00 & 13.82 & -3.95 & -4.64 \\
\hline TZ Mon & 0.871 & 0.44 & 11.92 & 10.79 & 9.47 & -0.12 & -1.99 & 13.16 & -3.83 & -4.57 \\
\hline W Sgr & 0.881 & 0.11 & 5.41 & 4.67 & 3.85 & -0.01 & -2.03 & 8.33 & -4.03 & -4.71 \\
\hline RX Cam & 0.898 & 0.57 & 8.88 & 7.68 & 6.26 & +0.03 & -2.05 & 9.89 & -4.09 & -4.77 \\
\hline W Gem & 0.898 & 0.28 & 7.87 & 6.95 & 5.97 & -0.04 & -1.84 & 10.26 & -4.24 & -4.85 \\
\hline U Vul & 0.903 & 0.65 & 8.41 & 7.13 & 5.61 & +0.05 & -2.05 & 9.11 & -4.14 & -4.80 \\
\hline DL Cas & 0.903 & 0.53 & 10.12 & 8.97 & 7.66 & -0.01 & -1.92 & 11.46 & -4.25 & -4.87 \\
\hline AC Mon & 0.904 & 0.51 & 11.28 & 10.10 & 8.71 & -0.22 & -2.04 & 12.41 & -3.99 & -4.72 \\
\hline S Sge & 0.923 & 0.13 & 6.42 & 5.61 & 4.78 & +0.10 & -1.84 & 9.37 & -4.18 & -4.85 \\
\hline TX Mon & 0.940 & 0.51 & 12.08 & 10.97 & 9.63 & -0.14 & -2.16 & 13.55 & -4.27 & -4.93 \\
\hline FN Aql & 0.977 & 0.51 & 9.62 & 8.38 & 7.00 & -0.02 & -1.80 & 10.93 & -4.24 & -4.95 \\
\hline SX Vel & 0.980 & 0.25 & 9.17 & 8.26 & 7.25 & -0.03 & -2.02 & 11.78 & -4.34 & -5.02 \\
\hline YZ Sgr & 0.980 & 0.29 & 8.38 & 7.34 & 6.21 & +0.05 & -1.86 & 10.55 & -4.18 & -4.92 \\
\hline S Nor & 0.989 & 0.19 & 7.37 & 6.43 & 5.42 & +0.05 & -1.86 & 9.97 & -4.17 & -4.93 \\
\hline$\beta$ Dor & 0.993 & 0.04 & 4.56 & 3.75 & 2.94 & -0.01 & -1.83 & 7.80 & -4.19 & -4.95 \\
\hline$\zeta$ Gem & 1.006 & 0.02 & 4.71 & 3.90 & 3.10 & +0.04 & -1.75 & 8.01 & -4.17 & -4.95 \\
\hline Z Lac & 1.037 & 0.40 & 9.51 & 8.42 & 7.20 & +0.01 & -1.93 & 11.59 & -4.51 & -5.20 \\
\hline VX Per & 1.037 & 0.52 & 10.46 & 9.31 & 8.00 & -0.05 & -1.98 & 12.25 & -4.65 & -5.28 \\
\hline AA Gem & 1.053 & 0.33 & 10.80 & 9.73 & 8.58 & -0.24 & -1.81 & 13.13 & -4.49 & -5.20 \\
\hline RX Aur & 1.065 & 0.28 & 8.63 & 7.67 & 6.66 & -0.07 & -1.89 & 11.46 & -4.69 & -5.35 \\
\hline HW Pup & 1.129 & 0.72 & 13.35 & 12.10 & 10.55 & -0.20 & -2.37 & 14.78 & -5.07 & -5.67 \\
\hline VY Sgr & 1.132 & 1.28 & 13.46 & 11.45 & 9.19 & +0.26 & -1.50 & 12.28 & -5.07 & -5.65 \\
\hline AD Pup & 1.133 & 0.33 & 10.94 & 9.90 & 8.71 & -0.24 & -2.12 & 13.49 & -4.68 & -5.43 \\
\hline BN Pup & 1.136 & 0.44 & 11.09 & 9.89 & 8.56 & +0.01 & -1.95 & 13.11 & -4.66 & -5.42 \\
\hline TT Aql & 1.138 & 0.50 & 8.43 & 7.13 & 5.72 & +0.11 & -1.73 & 10.15 & -4.65 & -5.41 \\
\hline
\end{tabular}


Table 10. continued.

\begin{tabular}{|c|c|c|c|c|c|c|c|c|c|c|}
\hline Name & $\log P$ & $\overline{E(B-V)}$ & $\bar{B}$ & $\bar{V}$ & $\bar{I}$ & $\begin{array}{r}{[\mathrm{Fe} / \mathrm{H}]_{A}} \\
\pm 0.10\end{array}$ & $\begin{array}{r}\log Z \\
\pm 0.15\end{array}$ & $\begin{array}{r}\mu_{0} \\
\pm 0.15\end{array}$ & $\begin{array}{r}M_{V} \\
\pm 0.15\end{array}$ & $\begin{array}{r}M_{I} \\
\pm 0.15\end{array}$ \\
\hline 1 & 2 & 3 & 4 & 5 & 6 & 7 & 8 & 9 & 10 & 11 \\
\hline UZ Sct & 1.169 & 1.07 & 13.15 & 11.25 & 9.18 & +0.33 & -1.37 & 12.68 & -4.96 & -5.63 \\
\hline SV Mon & 1.183 & 0.25 & 9.31 & 8.27 & 7.13 & 0.00 & -1.97 & 12.14 & -4.69 & -5.50 \\
\hline AV Sgr & 1.188 & 1.27 & 13.39 & 11.30 & 8.88 & +0.34 & -1.67 & 11.94 & -4.83 & -5.58 \\
\hline X Cyg & 1.214 & 0.29 & 7.53 & 6.39 & 5.24 & +0.12 & -1.68 & 10.29 & -4.85 & -5.63 \\
\hline CD Cyg & 1.232 & 0.51 & 10.25 & 8.95 & 7.50 & +0.07 & -1.93 & 12.19 & -4.94 & -5.71 \\
\hline SZ Aql & 1.234 & 0.64 & 10.06 & 8.63 & 7.07 & +0.15 & -1.76 & 11.57 & -5.05 & -5.78 \\
\hline RZ Vel & 1.310 & 0.34 & 8.21 & 7.08 & 5.86 & -0.07 & -2.01 & 11.14 & -5.17 & -5.96 \\
\hline V340 Ara & 1.318 & 0.57 & 11.76 & 10.21 & 8.58 & +0.31 & -1.52 & 13.23 & -4.92 & -5.80 \\
\hline WZ Sgr & 1.339 & 0.47 & 9.43 & 8.03 & 6.53 & +0.17 & -1.76 & 11.48 & -4.99 & -5.88 \\
\hline VZ Pup & 1.365 & 0.47 & 10.78 & 9.63 & 8.30 & -0.16 & -2.25 & 13.64 & -5.57 & -6.28 \\
\hline SW Vel & 1.370 & 0.35 & 9.27 & 8.12 & 6.84 & -0.07 & -2.13 & 12.24 & -5.28 & -6.10 \\
\hline X Pup & 1.414 & 0.44 & 9.73 & 8.53 & 7.17 & -0.03 & -2.16 & 12.62 & -5.56 & -6.33 \\
\hline T Mon & 1.432 & 0.21 & 7.29 & 6.13 & 4.98 & +0.13 & -1.67 & 10.77 & -5.33 & -6.20 \\
\hline RY Vel & 1.449 & 0.56 & 9.74 & 8.37 & 6.83 & -0.03 & -2.14 & 12.10 & -5.58 & -6.39 \\
\hline KQ Sco & 1.458 & 0.90 & 11.75 & 9.81 & 7.66 & +0.16 & -1.68 & 12.00 & -5.15 & -6.13 \\
\hline AQ Pup & 1.479 & 0.51 & 10.07 & 8.70 & 7.14 & -0.14 & -2.20 & 12.50 & -5.48 & -6.37 \\
\hline RS Pup & 1.617 & 0.45 & 8.46 & 7.03 & 5.49 & +0.17 & -1.98 & 11.31 & -5.74 & -6.70 \\
\hline SV Vul & 1.653 & 0.57 & 8.67 & 7.21 & 5.69 & +0.03 & -1.78 & 11.65 & -6.32 & -7.09 \\
\hline S Vul & 1.835 & 0.83 & 10.85 & 8.96 & 6.94 & -0.02 & -1.72 & 12.73 & -6.50 & -7.44 \\
\hline
\end{tabular}

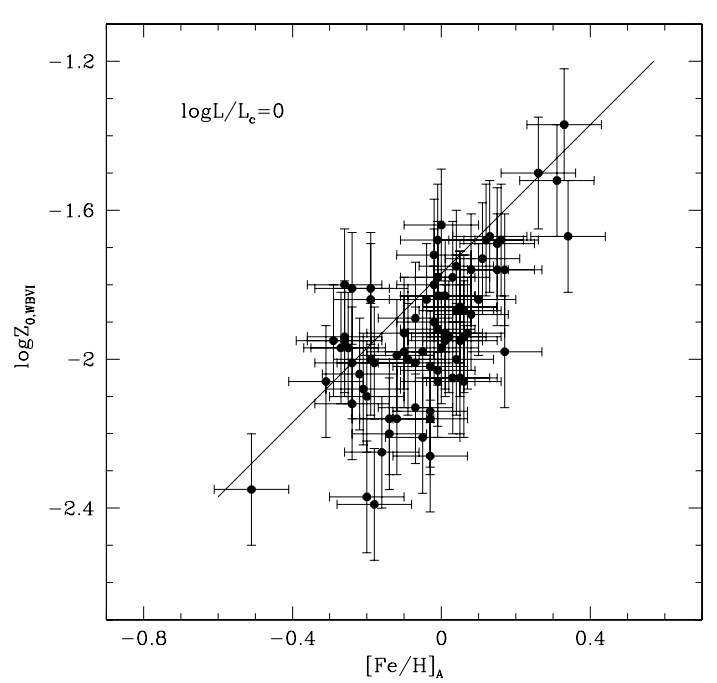

Fig. 11. Pulsation metal content of Galactic Cepheids determined by the predicted $P-W B V$ and $P-W V I$ relations versus the spectroscopic measurement $[\mathrm{Fe} / \mathrm{H}]_{A}$ by Andrievski and coworkers. The solid line is the relation $\log Z=[\mathrm{Fe} / \mathrm{H}]-1.77$ with $Z_{\odot}=0.017$.

whose slopes are closer to the B07 than to the S04 results and shallower than the LMC observed values listed in Table 11. Furthermore, we find that for the Cepheids with $[\mathrm{Fe} / \mathrm{H}] \leq 0$ the slope of the $P-M_{V}$ and $P-M_{I}$ relations is -2.69 and -2.94 , respectively, whereas for those with $[\mathrm{Fe} / \mathrm{H}] \geq 0$ we obtain -2.49 and -2.80 , further supporting the predicted dependence of the optical $P L$ relations slope on the metal content. We wish to emphasize the reliability of these results because they are independent of the $\log L / L_{\text {can }}$ ratio, which influences only the zero-point, and are based on the predicted evolutionary $P-W B V$ and $P-W V I$ relations whose slopes are the same as the LMC observed relations. We further discuss this issue at the end of this section.

\subsection{Magellanic Cloud Cepheids}

The BVI magnitudes of the many hundreds of LMC Cepheids collected during the OGLE-II micro-lensing survey

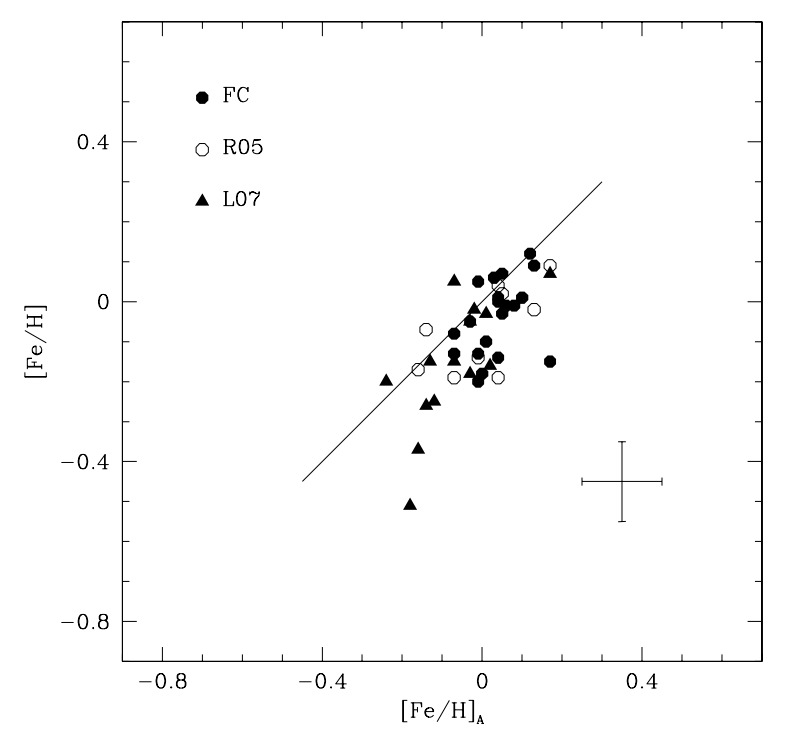

Fig. 12. Spectroscopic measurements by Fry \& Carney (1997: FC), Romaniello et al. (2005: R05) and Lemasle et al. (2007: L07) in comparison with the Andrievky and coworkers values. The solid line is the equality line and the large cross shows the average uncertainty of the spectroscopic data.

(Udalski et al. 1999) and the near-infrared $J K_{\mathrm{S}}$ measurements recently presented by $\mathrm{P} 04$ for 92 variables yield the apparent $P L$ and $P W$ relations listed in Table 11. The slopes of the observed $P L$ relations appear quite consistent with those predicted by the synthetic linear $P L$ relations at $Z=0.008$, the canonical metal content of LMC variables, and compared with those provided by the B07 Galactic Cepheids support the predicted steepening of the $P L$ relations when moving from metal-rich to metalpoor variables. Regarding the $P W$ relations, the observed slopes are also in close agreement with the predicted values listed in Table 3, excluding significant variation of $\log L / L_{\mathrm{can}}$ with the period among the LMC Cepheids. 


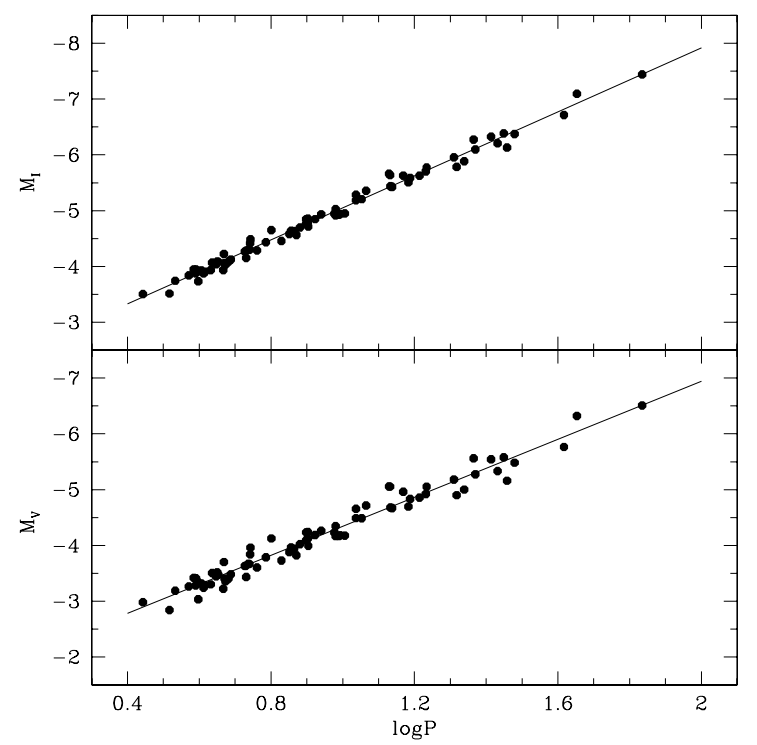

Fig. 13. $M_{V}$ and $M_{I}$ magnitudes versus period for Galactic Cepheids with spectroscopic $[\mathrm{Fe} / \mathrm{H}]_{A}$ measurements. The solid lines are drawn by a linear regression through the points.

Table 11. Apparent $P L$ and $P W$ relations for LMC Cepheids, as based on $V I$ magnitudes (Udalski et al. 1999) and 2Mass near-infrared magnitudes (P04).

\begin{tabular}{lccclccc}
\hline \hline$M_{i}$ & $\alpha$ & $\beta$ & $\sigma$ & $W$ & $\alpha$ & $\beta$ & $\sigma$ \\
\hline$B_{0}$ & 17.37 & -2.44 & 0.24 & $W B V$ & 16.01 & -3.83 & 0.22 \\
$V_{0}$ & 17.07 & -2.78 & 0.16 & $W V I$ & 15.88 & -3.29 & 0.08 \\
$I_{0}$ & 16.59 & -2.98 & 0.11 & & & & \\
$J_{0}$ & 16.34 & -3.15 & & & & & \\
$K_{s 0}$ & 16.04 & -3.26 & & $W J K_{s}$ & 15.90 & -3.36 & 0.05 \\
\hline
\end{tabular}

By comparison of the predicted evolutionary $P W$ relations with the observed $W B V, W V I$ and $W J K^{4}$ functions, we derive

$$
\begin{aligned}
& \mu_{0, W B V}=20.21( \pm 0.20)-0.72 \log L / L_{\mathrm{can}}+0.71 \log Z \\
& \mu_{0, W V I}=18.56( \pm 0.08)-0.84 \log L / L_{\mathrm{can}}-0.08 \log Z \\
& \mu_{0, W J K}=18.57( \pm 0.08)-0.90 \log L / L_{\mathrm{can}}-0.08 \log Z
\end{aligned}
$$

where the large uncertainty of the $W B V$-based distance reflects the observed dispersion around the $P-W B V$ relation which is likely to be due to less accurate sampling of the light curves and/or to a moderate metallicity spread among LMC Cepheids. In any case, our pulsation approach yields that the agreement among the $P W$-based distances is achieved at $Z \sim 0.008$, which is the classical metal content of LMC Cepheids. Using only the $W V I$ and $W J K$ functions which are mildly metallicity dependent, we find $\mu_{0}(\mathrm{LMC})=18.52 \pm 0.11 \mathrm{mag}$ at $Z=0.008$ and $\log L / L_{\text {can }}=0.25$, the average value suggested by the B07 Galactic Cepheids.

For the Magellanic Cepheids with ISB distances determined by $\mathrm{G} 05$, by repeating the procedure adopted for the B07 and S04 Galactic Cepheids, we derive the pulsation metal abundances and distances at $\log L / L_{\text {can }}=0$ and 0.25 listed in Cols. 4, 5 and 6,7 , respectively, in Table 12 . Initially, we find that at a constant $\log L / L_{\text {can }}$ ratio the pulsation distance to LMC Cepheids is independent of the period, at variance with the ISB results

${ }^{4}$ The 2 Mass $J K_{\mathrm{s}}$ magnitudes given by $\mathrm{P} 04$ are transformed into the Bessel \& Brett system adopting the relations given by Carpenter (2001). listed in Col. 3. On the other hand, taking at face value the ISBbased distance to derive the $\log L / L_{\text {can }}$ ratios listed in Col. 8 , we find $\log L / L_{\text {can }}=0.59-0.24 \log P$ which disagrees with the constant ratio already shown by the $P-W V I$ and $P-W J K$ relations of the OGLE and P04's variables. Interestingly enough, by comparing the ISB distances with the pulsation distance moduli at $\log L / L_{\text {can }}=0.25$, which is the average value of the B07 Galactic Cepheids, we derive

$$
\Delta \mu_{0}\left(p u l s-I S B_{\text {old }}\right)=0.29-0.20 \log P
$$

which is surprisingly similar to the G05 relation given in Eq. (3), but significantly different from the corresponding correction to the S04 Galactic Cepheids given in Eq. (5). In other words, our pulsation approach suggests that the G05 correction to ISBbased distances holds for LMC Cepheids but would lead to deceptive results (i.e., steeper $P L$ relations) if used for the (metalricher) Galactic Cepheids. Given the small number and the rather constant period of the SMC Cepheids with ISB distance, no firm conclusion can be reached for these metal-poorer variables.

Regarding the metal content inferred by the evolutionary $P W$ relations, the values at $\log L / L_{\text {can }}=0$ listed in Table 12 give $\log Z=-2.02 \pm 0.17$ and $-2.53 \pm 0.17$ for $\mathrm{LMC}$ and SMC Cepheids, respectively, with a fair decrease of 0.05 dex at $\log L / L_{\text {can }}=0.25$. These pulsation results agree with the widely known average spectroscopic values $[\mathrm{Fe} / \mathrm{H}] \sim-0.4(\mathrm{LMC})$ and -0.7 (SMC).

\subsection{Comments on the universality of the PL relations}

We have shown that the $H S T$-based distances to Galactic Cepheids support the predicted shallower slope of the $P-M_{V}$ and $P-M_{I}$ relations with respect to the LMC relations, at odds with other empirical studies supporting the universality of the $P L$ relations or, even worse, highlighting out that the Galactic relations are steeper than the LMC ones.

In particular, the results of the Araucaria Project (Pietrzynski et al. 2007, and references therein) dealing with Cepheid observations in Local Group galaxies suggest no significant change of the slope in the oxygen abundance ${ }^{5}$ range from $[\mathrm{O} / \mathrm{H}]=-0.4$ to $\sim-1.0$, i.e., for lower metal abundances than $Z=0.008$. This finding is not inconsistent with the theoretical $P-M_{V}$ slopes which vary by $10 \%$ from $Z=0.02$ to $Z=0.008$ but only $4 \%$ from $Z=0.008$ to $Z=0.004$, suggesting a sort of saturation in the metallicity effect at very low metal abundances. As for the $P-M_{I}$ relation, the predicted results already show no metallicity dependence below $Z=0.008$. We plan to compute new pulsation models at $Z \leq 0.002$ to verify this point.

Regarding the occurrence of steeper Galactic $P L$ relations, we have shown that the S04 and G05 results are biased by the adopted correction to ISB-based distances. On the other hand, other papers, e.g., Sandage et al. (2004) and Ngeow \& Kanbur (2004), adopt Cepheid average distances from different available measurements which may differ by up to $0.5 \mathrm{mag}$. Following a different approach to test the universality of the $P L$ and $P W$ relations, we adopt the observed $L M C$ relation $W V I=15.88-\mu_{0}(L M C)-3.29 \log P$ provided by the OGLE data to derive the intrinsic distance modulus to each Galactic Cepheid from its measured $W V I$ function. Once the apparent $V$ and $I$ magnitudes are corrected for reddening, this method provides the absolute Galactic $P M_{V}$ and $P M_{I}$ relations for each given LMC distance.

\footnotetext{
${ }^{5}[\mathrm{O} / \mathrm{H}]=\log (\mathrm{O} / \mathrm{H})-\log (\mathrm{O} / \mathrm{H})_{\odot}$, with $\log (\mathrm{O} / \mathrm{H})_{\odot}=-3.1$.
} 
Table 12. Magellanic Cloud Cepheids with ISB-based distance. From left to right, the columns give: name (1), period (2), ISB distance (3), pulsation metal content $\log Z$ and distance modulus $\mu_{0}$ at $\log L / L_{\mathrm{can}}=0$ [(4), (5)] and $\log L / L_{\mathrm{can}}=0.25$ [(6), (7)]. The last four columns give the final $\log L / L_{\text {can }}$ ratio and metal content derived by comparison with the ISB distance without [(8), (9)] or with [(10), (11)] the correction given by G05. In the last part of the table, we list the results for additional SMC Cepheids observed by Laney \& Stobie (1994).

\begin{tabular}{|c|c|c|c|c|c|c|c|c|c|c|}
\hline Name & $\log P$ & $\begin{array}{c}\mu_{0, I S B} \\
( \pm)\end{array}$ & $\begin{array}{l}\log Z \\
\pm 0.15\end{array}$ & $\begin{array}{c}\mu_{0, W} \\
\pm 0.15\end{array}$ & $\begin{array}{l}\log Z \\
\pm 0.15\end{array}$ & $\begin{array}{c}\mu_{0, W} \\
\pm 0.15\end{array}$ & $\begin{array}{c}\log L / L_{\mathrm{can}} \\
( \pm)\end{array}$ & $\begin{array}{l}\log Z \\
\pm 0.15\end{array}$ & $\begin{array}{c}\log L / L_{\mathrm{can}} \\
( \pm)\end{array}$ & $\begin{array}{c}\log Z \\
( \pm 0.15\end{array}$ \\
\hline 1 & 2 & 3 & 4 & 5 & 6 & 7 & 8 & 9 & 10 & 11 \\
\hline \multicolumn{11}{|l|}{$\overline{\mathrm{LMC}}$} \\
\hline HV12199 & 0.4215 & $18.336(0.094)$ & -2.05 & 18.66 & -2.09 & 18.44 & $+0.37(0.20)$ & -2.12 & +0.13 & -2.07 \\
\hline HV12203 & 0.4704 & $18.481(0.092)$ & -1.87 & 18.73 & -1.92 & 18.51 & $+0.29(0.20)$ & -1.93 & +0.05 & -1.88 \\
\hline HV12202 & 0.4915 & $18.289(0.072)$ & -1.82 & 18.63 & -1.87 & 18.41 & $+0.39(0.18)$ & -1.90 & +0.16 & -1.85 \\
\hline HV12197 & 0.4975 & $18.165(0.058)$ & -2.02 & 18.70 & -2.06 & 18.49 & $+0.62(0.18)$ & -2.14 & +0.39 & -2.09 \\
\hline HV12204 & 0.5364 & $18.202(0.044)$ & -2.19 & 18.67 & -2.24 & 18.46 & $+0.55(0.17)$ & -2.29 & +0.32 & -2.25 \\
\hline HV 12198 & 0.5469 & $18.314(0.028)$ & -1.98 & 18.73 & -2.03 & 18.51 & $+0.48(0.17)$ & -2.07 & +0.26 & -2.03 \\
\hline HV12816 & 0.9595 & $18.328(0.087)$ & -2.41 & 18.82 & -2.46 & 18.60 & $+0.57(0.19)$ & -2.52 & +0.43 & -2.50 \\
\hline HV12815 & 1.4169 & $18.296(0.028)$ & -1.86 & 18.58 & -1.91 & 18.36 & $+0.33(0.17)$ & -1.93 & +0.29 & -1.92 \\
\hline HV879 & 1.5662 & $18.532(0.040)$ & -1.97 & 18.76 & -2.02 & 18.55 & $+0.27(0.17)$ & -2.02 & +0.26 & -2.02 \\
\hline HV2338 & 1.6254 & $18.663(0.023)$ & -2.02 & 18.64 & -2.07 & 18.43 & $-0.02(0.17)$ & -2.01 & -0.02 & -2.01 \\
\hline \multicolumn{11}{|l|}{ SMC } \\
\hline HV1345 & 1.1296 & $18.828(0.081)$ & -2.46 & 19.24 & -2.51 & 19.02 & $+0.48(0.19)$ & -2.56 & +0.38 & -2.54 \\
\hline HV1335 & 1.1578 & $18.875(0.082)$ & -2.59 & 19.46 & -2.64 & 19.25 & $+0.68(0.19)$ & -2.72 & +0.59 & -2.70 \\
\hline HV1328 & 1.1996 & $18.732(0.087)$ & -2.56 & 19.21 & -2.61 & 19.00 & $+0.56(0.19)$ & -2.67 & +0.48 & -2.66 \\
\hline HV1333 & 1.2120 & $19.388(0.080)$ & -2.45 & 19.45 & -2.50 & 19.23 & $+0.07(0.19)$ & -2.47 & -0.01 & -2.45 \\
\hline HV822 & 1.2238 & $19.091(0.081)$ & -2.24 & 19.27 & -2.29 & 19.05 & $+0.20(0.19)$ & -2.28 & +0.12 & -2.27 \\
\hline HV1365 & 1.0940 & & -2.39 & 19.50 & -2.44 & 19.28 & & & & \\
\hline HV1954 & 1.2230 & & -2.70 & 18.89 & -2.75 & 18.67 & & & & \\
\hline HV817 & 1.2770 & & -2.60 & 19.05 & -2.65 & 18.83 & & & & \\
\hline HV11211 & 1.3300 & & -2.34 & 18.89 & -2.40 & 18.67 & & & & \\
\hline HV2209 & 1.3550 & & -2.67 & 19.03 & -2.72 & 18.81 & & & & \\
\hline HV847 & 1.4330 & & -2.35 & 19.23 & -2.40 & 19.01 & & & & \\
\hline HV823 & 1.5040 & & -2.17 & 19.18 & -2.22 & 18.96 & & & & \\
\hline HV865 & 1.5230 & & -2.74 & 18.96 & -2.80 & 18.74 & & & & \\
\hline HV2064 & 1.5270 & & -2.32 & 19.32 & -2.38 & 19.10 & & & & \\
\hline HV2195 & 1.6210 & & -2.50 & 19.12 & -2.56 & 18.90 & & & & \\
\hline HV824 & 1.8180 & & -2.54 & 19.01 & -2.60 & 18.80 & & & & \\
\hline
\end{tabular}

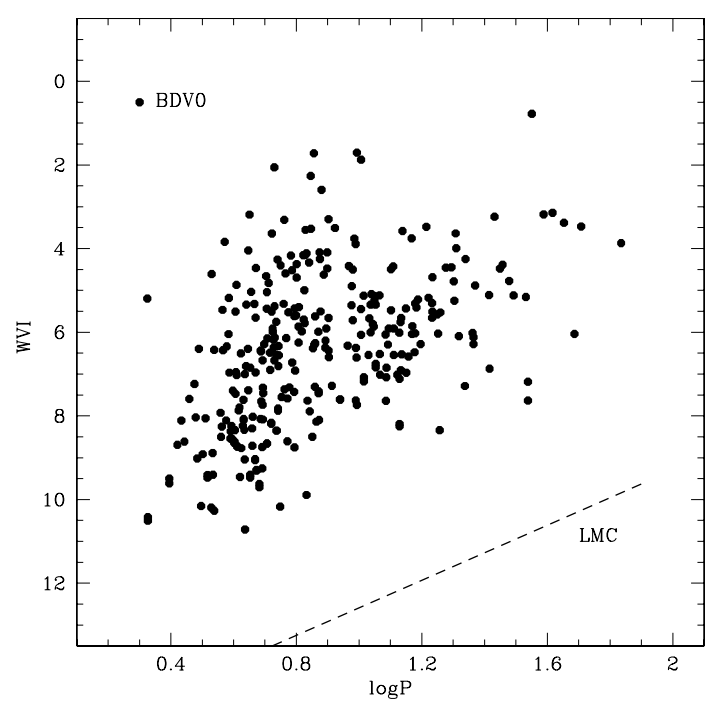

Fig. 14. Observed $W V I$ functions versus period for fundamental Galactic Cepheids with measured $V$ and $I$ magnitudes compiled by Berdnikov et al. (2000, BDV0). The dashed line is the observed LMC Cepheid relation.

Figure 14 shows all the fundamental Galactic Cepheids listed by Berdnikov et al. (2000) in comparison with the observed $P$-WVI relation of LMC Cepheids. Adopting the $E(B-V)$ reddening listed by Fernie et al. (1995), we show in Fig. 15 that the resulting relations (solid lines) are

$$
\begin{aligned}
& M_{V}=17.14( \pm 0.17)-\mu_{0}(L M C)-2.56( \pm 0.17) \log P \\
& M_{I}=16.71( \pm 0.12)-\mu_{0}(L M C)-2.83( \pm 0.10) \log P
\end{aligned}
$$

This result clearly demonstrates that assuming that the LMC Wesenheit relations are universally valid lead to absolute Galactic $P M_{V}$ and $P M_{I}$ relations whose slopes are significantly different from the LMC slopes. In particular, the Galactic relations are not only shallower than the $L M C P M_{V}$ and $P M_{I}$ relations (dashed lines) given in Table 11, but even fainter (at $\log P \geq 0.5$ ), irrespective of the LMC distance.

\section{Conclusions}

We have computed new sets of nonlinear fundamental pulsation models for classical Cepheids assuming four different chemical compositions $(Z=0.004,0.008,0.01$ and 0.02$)$ and adopting an increased value $\left(l / H_{\mathrm{p}}=1.7-1.8\right)$ of the mixing-length parameter used in the hydrodynamical code to close the system of nonlinear equations. By combining the results of these calculations with our previously computed models at $l / H_{\mathrm{p}}=1.5 \mathrm{we}$ find that:

1. increasing the $l / H_{\mathrm{p}}$ value the Cepheid instability strip gets narrower and the effect is greater at the higher metal contents $(Z \geq 0.01)$. However, the synthetic period-magnitude 


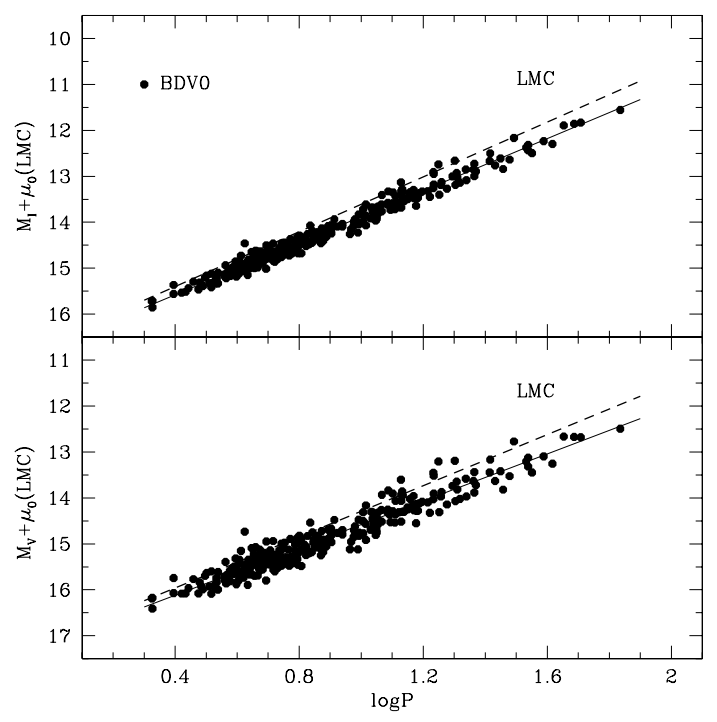

Fig. 15. $M_{V}$ and $M_{I}$ magnitudes versus period for the Galactic Cepheids in Fig. 14. The solid lines are drawn by a linear regression through the points, while the dashed lines are the LMC Cepheid relation.

relations based on the combination of pulsation results with updated evolutionary models are slightly affected by the adopted $l / H_{\mathrm{p}}$ value and confirm the already shown dependence of the slope, the zero-point and the intrinsic dispersion on the wavelength and the adopted metallicity;

2. by using all the fundamental models computed with different choices of the metal and helium abundance, we determine mass-dependent period-Wesenheit relations which are useful to estimate the actual mass of Cepheids of known distance and metal content;

3. to avoid any assumption on the Cepheid mass-luminosity relation, we introduce the ratio $\log L / L_{\text {can }}$, where $L_{\text {can }}$ is the luminosity inferred by canonical (no mass-loss, no convective core overshooting) evolutionary computations, and we derive evolutionary period-Wesenheit relations which provide the distance to Cepheids with known metal content, once the $L / L_{\text {can }}$ ratio is adopted. As discussed in the text, the release of the canonical evolutionary frame yields positive $\log L / L_{\text {can }}$ values;

4. we show that the effects of $l / H_{\mathrm{p}}$ (at fixed $Z$ and $Y$ ) and $Y$ (at fixed $l / H_{\mathrm{p}}$ and $Z$ ) on the evolutionary period-Wesenheit relations are quite negligible. In the meanwhile, we show that the predicted $P-W B V$ relation has an opposite metallicity dependence with respect to $P-W V I$ and $P-W V K$, suggesting a quite plain method to derive the distance and metallicity of observed variables, independent of reddening.

By comparing of the theoretical relations with Galactic and LMC Cepheids, we derive the following results:

1. the pulsation distances inferred from the predicted evolutionary $P W$ relations are consistent with the recent $H S T$ astrometric measurements given by Benedict et al. (2007) for a sample of Galactic Cepheids and suggest an average value of $\log L / L_{\text {can }}=0.25 \pm 0.12$ or a mild period-dependence as $\log L / L_{\mathrm{can}}=0.41-0.19 \log P$;

2 . on the contrary, the pulsation distances disagree with those determined by Storm et al. (2004) for Galactic Cepheids, as based on the Infrared Surface Brightness technique, unless negative (unrealistic) $\log L / L_{\text {can }}$ ratios and $\log L / L_{\text {can }}=$ $0.85-0.55 \log P$ are adopted;
3. adopting for these variables $\log L / L_{\mathrm{can}}=0.25$, the average value inferred from the Benedict et al. (2007) Cepheids, we determine a period-dependent correction to the ISB-based distances as given by

$\Delta \mu_{0}\left(p u l s-I S B_{\text {old }}\right)=0.50-0.45 \log P$,

which is significantly different from the correction

$\Delta \mu_{0}\left(I S B_{\text {new }}-I S B_{\text {old }}\right)=0.29-0.18 \log P$

determined by Gieren et al. (2005) to remove the puzzling dependence on the period of the ISB distances to LMC Cepheids. It is of interest to note that applying our pulsation approach to these LMC Cepheids, we actually obtain

$\Delta \mu_{0}\left(p u l s-I S B_{\text {old }}\right)=0.29-0.20 \log P$,

suggesting an additional metallicity effect on the perioddependent correction to ISB distances;

4. the reliability of the pulsation method to infer the Cepheid metallicity is verified by comparison with available spectroscopic data for Galactic and LMC Cepheids;

5. the slopes of the observed period-magnitude relations of the hundreds of LMC Cepheids observed by Udalski et al. (1999) and Persson et al. (2004) are in close agreement with the those predicted at $Z=0.008$. A similar agreement is found for the period-Wesenheit relations, which also exclude a significant variation of the $L / L_{\text {can }}$ ratio among the LMC Cepheids;

6. the comparison of predicted with observed $P-W V I$ and $P$ $W J K$ relations gives $\mu_{0}(\mathrm{LMC})=18.73 \pm 0.09 \mathrm{mag}$ at $\log L / L_{\text {can }}=0$ and $18.52 \pm 0.11 \mathrm{mag}$ at $\log L / L_{\text {can }}=0.25$, the average value suggested by the B07 Galactic Cepheids;

7. the predicted steepening of the optical period-magnitude relations when passing from metal-rich to metal-poor variables is supported by the $H S T$-based distances to Galactic Cepheids, as well as by observational evidence based on the straight application to Galactic Cepheids of the empirical LMC $P-W V I$ relation. Moreover, we show that at $\log P \geq$ 0.5 the Galactic $P-M_{V}$ and $P-M_{I}$ relations are fainter than LMC relations in agreement with the theoretical predictions.

Acknowledgements. We thank the anonymous referee for several valuable comments and suggestions. It is a pleasure to acknowledge useful conversations with Michele Cignoni and Giuseppe Bono. Financial support for this study was provided by MIUR, under the scientific project "Continuity and Discontinuity in the Milky Way Formation” (PI: Raffaele Gratton).

\section{References}

Alcock, C., Allsman, R. A., Axelrod, T. S., et al. 1995, AJ, 109, 1653 Alibert, Y., Baraffe, I., Hauschildt, P., \& Allard, F. 1999, A\&A, 344, 551 Andrievsky, S. M., Kovtyukh, V. V., \& Luck, R. E. 2002a, A\&A, 381, 32 Andrievsky, S. M., Bersier, D., \& Kovtyukh, V. V. 2002b, A\&A, 384, 140 Andrievsky, S. M., Kovtyukh, V. V., \& Luck, R. E. 2002c, A\&A, 392, 491 Andrievsky, S. M., Luck, R. E., Martin, P., \& Lépine, J. R. D. 2004, A\&A, 413, 159

Antonello, E., Poretti, E., \& Reduzzi, L. 1990, A\&A, 236, 138

Baraffe, I., \& Alibert, Y. 2001, A\&A, 371, 592

Beaulieu, J. P., Buchler, J. R., \& Kolláth, Z. 2001, A\&A, 380, 168

Benedict, G. F., McArthur, B. E., Feast, M. W., et al. 2007, AJ, 133, 181 [B07] Berdnikov, L. N., Dambis, A. K., \& Vozyakova, O. V. 2000, A\&AS, 143, 211 Bessell, M. S., \& Brett, J. M. 1988, PASP, 100, 113

Bono, G., \& Stellingwerf, M. 1994, ApJS, 93, 233

Bono, G., Marconi, M., \& Stellingwerf, R. F. 1999a, ApJS, 122, 167 (Paper I) Bono, G., Caputo, F., Castellani, V., \& Marconi, M. 1999b, ApJ, 512, 711 (Paper II)

Bono, G., Castellani, V., \& Marconi, M. 2000a, ApJ, 532, L129

Bono, G., Castellani, V., \& Marconi, M. 2000b, ApJ, 529, 293 (Paper III) 
Bono, G., Caputo, F.,Cassisi, S., et al. 2000c, ApJ, 543, 955 (B00)

Bono, G., Marconi, M., \& Stellingwerf, R. F. 2000d, A\&A, 360, 245 (Paper VI)

Bono, G., Gieren, W. P., Marconi, M., Fouqué, P., \& Caputo, F. 2001, ApJ, 563, 319 (B01)

Bono, G., Castellani,V., \& Marconi, M. 2002, ApJ, 565, L83

Caputo, F., Marconi, M., \& Musella, I. 2000, A\&A, 354, 610 (Paper V)

Carpenter, J. M. 2001, AJ, 121, 2851

Castellani, V., \& degl'Innocenti, S. 1995, A\&A, 298, 827

Castellani, V., Chieffi, A., \& Straniero, O. 1992, ApJS, 78, 517 (CCS)

Castellani, V., degl'Innocenti, S., \& Marconi, M. 2002, ASP Conf. Ser., 265, 193

Castellani, V., Degl'Innocenti, S., Marconi, M., Prada Moroni, P. G., \& Sestito, P. 2003, A\&A, 404, 645

Castelli, F., Gratton, R. G., \& Kurucz, R. L. 1997a, A\&A, 318, 841

Castelli, F., Gratton, R. G., \& Kurucz, R. L. 1997b, A\&A, 324, 432

Chiosi, C., Wood, P. R., \& Capitanio, N. 1993, ApJS, 86, 541

Cioni, M.-R. L., Girardi, L., Marigo, P., \& Habing, H. J. 2006, A\&A, 456, 967

Cordier, D., Goupil, M. J., \& Lebreton, Y. 2003, A\&A, 409, 491

Dean, J. F., Warren, P. R., \& Cousins, A. W. J. 1978, MNRAS, 183, 569

Di Criscienzo, M., Marconi, M., \& Caputo, F. 2004a, ApJ, 612, 1092

Di Criscienzo, M., Marconi, M., \& Caputo, F. 2004b, MmSAI, 75, 190

Fernie, J. D., Evans, N. R., Beattie, B., \& Seager, S. 1995, Inf. Bull. Variable Stars, 4148,1

Fiorentino, G., Caputo, F., Marconi, M., \& Musella, I. 2002, ApJ, 576, 402 (Paper VIII)

Fry, A. M., \& Carney, B. W. 1997, AJ, 113, 1073 (FC)

Gieren, W., Storm, J., Barnes, T. G., et al. 2005, ApJ, 627, 224G (G05)

Girardi, L., Bressan, A., Bertelli, G., \& Chiosi, C. 2000, A\&AS, 141, 371

Groenewegen, M. A. T., Romaniello, M., Primas, F., \& Mottini, M. 2004, A\&A, 420,655

Kanbur, S. M., Ngeow, C., Nikolaev, S., Tanvir, N. R., \& Hendry, M. A. 2003, A\&A, 411,361

Kennicutt, R. C., Jr., Stetson, P. B., \& Saha, A. 1998, ApJ, 498, 181

Kennicutt, R. C., Jr., Bresolin, F., \& Garnett, D. R. 2003, PASP, 115, 928

Kienzle, F., Moskalik, P., Bersier, D., \& Pont, F. 1999, A\&A, 341, 818
Laney, C. D. \& Stobie, R. S. 1993, MNRAS, 263, 921

Laney, C. D., \& Stobie, R. S. 1994, MNRAS, 266, 441

Lemasle, B., Francois, P., Bono, G., et al. 2007, A\&A, 467, 283 [L07]

Luck, R. E., Gieren, W. P., Andrievsky, S. M., et al. 2003, A\&A, 401, 939

Macri, L. M., Stanek, K. Z., Bersier, D., Greenhill, L. J., \& Reid, M. J. 2006, ApJ, 652, 1133

Madore, B. 1982, PASP, 94, 40

Madore, B., \& Freedman, W. L. 1991, PASP, 103, 933

Marconi, M., \& Clementini, G. 2005, AJ, 129, 2257

Marconi, M., Caputo, F., Di Criscienzo, M., \& Castellani, M. 2003, ApJ, 596, 299

Marconi, M., Bono, G., \& Nordgren, T. E. 2004, ASP Conf. Proc., 310, 502 (M04)

Marconi, M., Musella, I., \& Fiorentino, G. 2005, ApJ, 632, 590 (Paper IX)

Ngeow, C.-C., \& Kanbur, S. M. 2004, MNRAS, 349, 1130

Ngeow, C.-C., \& Kanbur, S. M. 2005, MNRAS, 360, 1033

Ngeow, C.-C., \& Kanbur, S. M. 2006, ApJ, 650, 180

Ngeow, C.-C., Kanbur, S. M., Nikolaev, S., et al. 2005, MNRAS, 363, 831

Persson, S. E., Madore, B. F., Krzeminski, W., et al. 2004, AJ, 128, 2239 (P04)

Pietrinferni, A., Cassisi, S., Salaris, M., \& Castelli, F. 2004, ApJ, 612, 168

Pietrzynski, G., Gieren, W., Udalski, A., et al. 2007, AJ, in press [arXiv:0704.2075v1]

Romaniello, M., Primas, F., Mottini, et al. 2005, A\&A, 429, 37 (R05)

Sakai, S., Ferrarese, L., Kennicutt, R. C., Jr., \& Saha, A. 2004, ApJ, 608, 42

Sandage, A., Bell, R. A., \& Tripicco, M. J. 1999, ApJ, 522, 250

Sandage, A., Tammann, G. A., \& Reindl, B. 2004, A\&A, 424, 43

Sasselov, D.D., Beaulieu, J. P., \& Renault, C. 1997, A\&A, 324, 471

Stellingwerf, R. F. 1982, ApJ, 262, 330S

Storm, J., Carney, B. W., Gieren, W. P., et al. 2004, A\&A, 415, 531 (S04)

Tammann, G.A., \& Reindl, B. 2002, Ap\&SS, 280, 165

Tammann, G.A., Sandage, A., \& Reindl, B. 2003, A\&A, 404, 423

Tanvir, N. R. 1999, ASP Conf. Ser., 167, 84

Udalski, A., Soszynski, I., Szymanski, M., et al. 1999, AcA, 49, 223 